\title{
Data report: elemental and isotopic composition and Rock- Eval pyrolysis of bulk sediments, IODP Expedition $344^{1}$
}

\author{
Ji-Hoon Kim, ${ }^{2}$ Jiyoung Choi, ${ }^{2}$ Gil-Young Kim, ${ }^{2}$ and Se-Won Chang ${ }^{2}$
}

\section{Chapter contents}

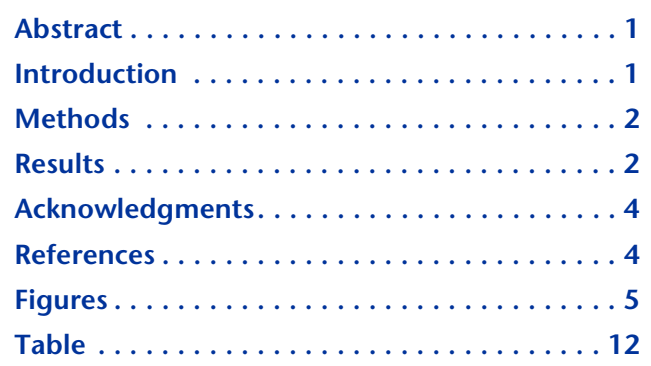

${ }^{1}$ Kim, J.-H., Choi, J., Kim, G.-Y., and Chang, S.-W., 2016. Data report: elemental and isotopic composition and Rock-Eval pyrolysis of bulk sediments, IODP Expedition 344. In Harris, R.N., Sakaguchi, A., Petronotis, K., and the Expedition 344 Scientists, Proceedings of the Integrated Ocean Drilling Program, 344: College Station, TX (Integrated Ocean Drilling Program). doi:10.2204/iodp.proc.344.204.2016 2 Petroleum and Marine Research Division, Korea Institute of Geoscience and Mineral Resources, 124 Gwahang-no, Yuseong-gu, Daejeon 34132, Republic of Korea. Correspondence author: jychoi@kigam.re.kr

\begin{abstract}
Multiple geochemical analyses (total carbon [TC], total nitrogen [TN], total sulfur, isotopic compositions of $\delta^{13} \mathrm{C}_{\text {org }}$ and $\delta^{15} \mathrm{~N}_{\text {org }}$, and Rock-Eval pyrolysis) were performed on bulk sediment samples from Integrated Ocean Drilling Program Expedition 344 to assess geochemical characteristics and variations with depth at each drilled site. Downcore profiles of geochemical analyses vary with site and composition and do not correlate with lithostratigraphic units. In addition, the downcore profiles of TC, total organic carbon (TOC), total inorganic carbon (TIC), and TN analyzed postcruise displayed similar trends to those determined from onboard analyses at all sites.

Based on Rock-Eval pyrolysis, most of the organic matter is at an immature stage of Type III (vascular land plants) evolution, which is inconsistent with the results from TOC/TN, $\delta^{13} \mathrm{C}_{\text {org }}$, and $\delta^{15} \mathrm{~N}_{\text {org }}$ that indicated that most of the organic matter originates from an admixture of marine and freshwater algae (Sites U1380 and U1413) or from marine algae (Site U1412).
\end{abstract}

\section{Introduction}

Integrated Ocean Drilling Program (IODP) Expedition 344 drilled five sites located offshore the Osa Peninsula, Costa Rica, where the incoming Cocos Ridge has lifted the seismogenic zone to within reach of scientific drilling (see the "Expedition 344 summary" chapter [Harris et al., 2013]). The location of Site U1381 is $\sim 4.5 \mathrm{~km}$ seaward of the deformation front offshore Osa Peninsula and Caño Island; Sites U1413, U1380, and U1412 are in the upper, middle, and base slope of the Costa Rica margin, respectively; and Site U1414 is on the flank of the subducting aseismic Cocos Ridge (see the "Expedition 344 summary" chapter [Harris et al., 2013]). Among the five sites, we selected Sites U1380, U1412, U1413, and U1414 to investigate the elemental and isotopic composition (total carbon [TC], total nitrogen [TN], total sulfur [TS], $\delta^{13} \mathrm{C}_{\text {org }}$, and $\delta^{15} \mathrm{~N}_{\text {org }}$ ) and Rock-Eval pyrolysis of sediment at erosional subduction zones. Based on lithostratigraphic units defined during the expedition, Site U1380 is divided into three units (Unit I and Subunits IIA and IIB), Sites U1412 and U1413 are divided into three units (Units I, II, and III), and Site U1414 is divided into five units (Subunits IA, IB, IIA, and IIB and Unit III) (see the "Expedition 344 summary" chapter [Harris et al., 
2013]). Correlation of elements and organic matter in bulk sediment with lithostratigraphic units can be useful for documenting the depositional environment and origin of organic matter. Hence, this study reports several geochemical results on bulk sediment from offshore the Osa Peninsula.

\section{Methods}

\section{Elemental analysis}

A total of 214 squeeze cake samples from Sites U1380, U1412, U1413, and U1414 were used for geochemical analyses. A part of each squeeze cake was dried using a freeze dryer for $24 \mathrm{~h}$ at the Korea Institute of Geoscience and Mineral Resources (KIGAM). After the bulk sediment samples were dried, they were ground and homogenized in an agate mortar. TC and TN contents were measured by combustion method using a CHN-900 (LECO, USA) apparatus at KIGAM with detection limits of $0.001 \%$ for TC and $0.01 \%$ for $\mathrm{TN}$. The reproducibility of TC and TN measurements, estimated from repeated analyses of the LECO soil standard $(\mathrm{TC}=2.6 \mathrm{wt} \%$ and $\mathrm{TN}=$ $0.209 \mathrm{wt} \%$ ) was $<3 \%$ and $<4 \%$, respectively. TS contents were determined using an S-144DR (LECO, USA) at KIGAM with a detection limit of $0.001 \%$. Four LECO standards $(0.28,0.95,1.51$, and 2.30 $\mathrm{wt} \%)$ were used for the analysis of TS. The reproducibility of TS was monitored by repeated analysis of LECO standard $(1.51 \mathrm{wt} \%)$ was $<3 \%$.

\section{Rock-Eval pyrolysis}

A Rock-Eval Turbo 6 (Vinci Technologies, France) instrument at KIGAM was utilized for the Rock-Eval pyrolysis. Free and adsorbed hydrocarbons released during programmed heating of a sample are recorded as the first peak in a pyrogram $\left(\mathrm{S}_{1}\right)$ under low temperature $\left(<300^{\circ} \mathrm{C}\right)$. The second peak $\left(\mathrm{S}_{2}\right)$ in the pyrogram represents hydrocarbons released by kerogen cracking when the sample is heated from $300^{\circ} \mathrm{C}$ to $650^{\circ} \mathrm{C}$. The maximum $\mathrm{S}_{2}$ temperature is defined as $T_{\max } . \mathrm{CO}_{2}$, shown as the third peak $\left(\mathrm{S}_{3}\right)$ in the program, is also generated by kerogen degradation. When these components are normalized to the total organic carbon (TOC) content, the $S_{2}$ peak becomes the hydrogen index $\left(\mathrm{HI}=\mathrm{S}_{2} \times 100 / \mathrm{TOC}\right)$ and $\mathrm{S}_{3}$ becomes the oxygen index $\left(\mathrm{OI}=\mathrm{S}_{3} \times 100 / \mathrm{TOC}\right)$ (Tissot and Welte, 1984; Peters, 1986). TOC was also measured by the Rock-Eval Turbo 6, summing up the pyrolyzed carbon (PC) and residual carbon (RC) (Arthur et al., 1998; Lafargue et al., 1998; Kim et al., 2007, 2014). IFP 160,000 (Vinci-Technologies, France) was used as standard for the Rock-Eval analysis. The re- productivity of TOC, $\mathrm{S}_{2}, \mathrm{~S}_{3}$, and $T_{\max }$ is estimated from repeated analyses of the IFP 160,000 standard was better than $2 \%, 4 \%, 5 \%$, and $1 \%$, respectively.

Total inorganic carbon (TIC) was calculated as the difference between TC measured by the CHN-900 and TOC measured by the Rock-Eval Turbo 6:

$$
\text { TIC }(w t \%)=\text { TC }(w t \%)-\text { TOC }(w t \%) .
$$

\section{Isotope analysis}

The aliquots of powdered bulk samples from Sites U1380, U1412, and U1413 were pretreated with $3 \mathrm{M}$ $\mathrm{HCl}$ to remove carbonate $\left(\mathrm{CaCO}_{3}\right)$ and inorganic nitrogen for the analysis of organic carbon $\left(\delta^{13} \mathrm{C}_{\text {org }}\right)$ and nitrogen $\left(\delta^{15} \mathrm{~N}_{\text {org }}\right.$ ) isotope ratios (Kim et al., 2014). $\delta^{13} \mathrm{C}_{\text {org }}$ and $\delta^{15} \mathrm{~N}_{\text {org }}$ were measured using a stable isotope ratio mass spectrometer system with elemental analyzer (Vision-EA, Isoprime, UK) at the National Instrumentation Center for Environmental Management (NICEM, Korea). Analytical reproducibility was $\pm 0.1 \%$ o for $\delta^{13} \mathrm{C}_{\text {org }}$ and $\pm 0.2 \%$ for $\delta^{15} \mathrm{~N}_{\text {org }}$. All carbon and nitrogen isotopes are reported in the usual $\delta$ notation relative to Vienna Peedee belemnite (VPDB) for carbon and atmospheric $\mathrm{N}_{2}$ for nitrogen:

$$
\delta(\%)=\left[\left(R_{\text {sample }}-R_{\text {standard }}\right) / R_{\text {standard }}\right] \times 1000,
$$

where $R$ represents the ${ }^{13} \mathrm{C} /{ }^{12} \mathrm{C}$ ratio and ${ }^{15} \mathrm{~N} /{ }^{14} \mathrm{~N}$ ratio of the sample and standard for each isotope.

\section{Results}

\section{Elemental composition}

The elemental composition of samples analyzed are listed in Table T1 and their downcore profiles are illustrated in Figures F1, F2, F3, and F4. Because not all samples from Sites U1380, U1412, and U1413 were analyzed for TC, TOC, and TN during the expedition, we completed the analyses of all samples from Expedition 344 postcruise. Most of the samples that were analyzed for TC, TOC, TIC, and TN content both during the expedition and postcruise display similar values at all sites. However, TOC content values obtained onboard from samples deeper than 160 mbsf at Site U1414 are higher than those measured postcruise (Fig. F4).

Downcore profiles of TC, TOC, TIC, and TN vary with depth at Site U1414 (Fig. F4). TOC and TN gradually decrease from the seafloor to $\sim 200 \mathrm{mbsf}$ and then slightly increase deeper than $270 \mathrm{mbsf}$, whereas TC and TIC contents are relatively constant from the seafloor to $\sim 120$ mbsf and then gradually increase 
with depth with the exception of the interval from 215 to 230 mbsf. Deeper than 120 mbsf, the downcore profiles of $\mathrm{TOC}$ and $\mathrm{TN}$ are mirror images to those of TC and TIC (Fig. F4). TOC also decreases from seafloor to $\sim 120 \mathrm{mbsf}$, with a minimum $(<1.0$ wt\%) deeper than $\sim 500$ mbsf at Site U1413 (Fig. F3). In contrast, downcore profiles of TC, TOC, TIC, and TN from seafloor to 490 mbsf at Sites U1380 and U1412 and downcore profiles of TC, TIC, and TN at Site U1413 do not show variation with depth (Table T1; Figs. F1, F2, F3).

Organic matter derived from marine algae typically has an atomic TOC/TN ratio of 4-10; ratios derived from vascular land plants are 20 or higher (Emerson and Hedges, 1988; Meyers, 1994). Most atomic TOC/ TN ratios at Sites U1380, U1412, and U1413 are 4-12 and are relatively constant with depth (Table T1; Figs. F1, F2, F3). On the other hand, TOC/TN ratios at Site U1414 are constant at 7-10 in the upper 200 mbsf and are mostly $>10$ deeper than 200 mbsf, varying from 9 to 38 (Table T1; Fig. F4). Deeper than 200 mbsf at Site U1414, TOC/TN ratios calculated using onboard and postcruise data show significant deviation because TOC contents are remarkably different between them (Fig. F4).

Most TS contents are $<3 \mathrm{wt} \%$ and have a mid-maximum around 50 mbsf at Site U1414 (Table T1; Fig. F4). In addition, TS values decrease with depth at Site U1380 (Table T1; Fig. F1). TOC/TS ratios at Sites U1380 and U1412 have maximum values in the lower sections ( $\sim 584 \mathrm{mbsf}$ at Site U1380 and $\sim 352$ mbsf at U1412), where TS content is at minimum values at each site (Table T1; Figs. F1, F2).

\section{Rock-Eval pyrolysis}

Most $\mathrm{S}_{2}$ and $\mathrm{S}_{3}$ values are $<2 \mathrm{mg} \mathrm{HC} / \mathrm{g}$ Rock and $<3$ $\mathrm{mg} \mathrm{CO} / \mathrm{g}$ Rock, respectively, and show higher values at shallow depths at Sites U1412-U1414 (Table T1; Figs. F1, F2, F3, F4). In addition, $S_{2}$ and $S_{3}$ values display mirror images to each other at depths shallower than 100 mbsf at Site U1412, whereas these values tend to decrease with depth deeper than $200 \mathrm{mbsf}$ at Site U1414 (Figs. F2, F4). $S_{3}$ values at Site U1413 also decrease with depth (Fig. F3).

Most $\mathrm{HI}$ and OI values range from 50 to $150 \mathrm{mg} \mathrm{HC/}$ $\mathrm{g}$ TOC and from 100 to $300 \mathrm{mg} \mathrm{CO} / \mathrm{g}$ TOC, respectively (Table T1; Figs. F1, F2, F3, F4). OI maximum values are found at $\sim 160$ mbsf at Site U1412, 110 mbsf at Site U1413, and 200 mbsf at Site U1414. Maximum OI values are in lithostratigraphic unit Unit I at Site U1412, Unit II at Site U1413, and at the
Subunit IIA/IIB boundary at Site U1414 (Figs. F2, F3, and F4).

Plots of modified van Krevelen-type and $S_{2}$ versus TOC diagrams using Rock-Eval data show that most data correspond to the Type III evolution field (Fig. F5). In addition, there is a strong positive correlation between $\mathrm{S}_{2}$ and TOC at Sites U1380, U1413, and U1414 $\left(R^{2}>0.80\right)$ and a moderate positive correlation at Site U1412 $\left(R^{2}=0.68\right)$ (Fig. F5).

$T_{\max }$ values are mostly lower than $435^{\circ} \mathrm{C}$, suggesting that organic matter is at a thermally immature stage (Nali et al., 2000). $T_{\max }$ generally does not show vertical variation with depth at Sites U1380, U1412, U1413, and U1414 (Table T1; Figs. F1, F2, F3, F4). However, $T_{\max }$ values rapidly decrease $\left(<400^{\circ} \mathrm{C}\right)$ between 175 and 200 mbsf in Subunit IIA at Site U1414 (Fig. F4).

\section{Isotopic composition}

$\delta^{13} \mathrm{C}_{\text {org }}$ and $\delta^{15} \mathrm{~N}_{\text {org }}$ values at Site U1380 display significant different trends at depths shallower than 500 mbsf (Table T1; Fig. F4). Their values at shallow depths are relatively constant $\left(\delta^{13} \mathrm{C}_{\text {org }}=27.01 \%\right.$ \pm $0.48 \%$ and $\delta^{15} \mathrm{~N}_{\text {org }}=6.70 \% 0 \pm 0.21 \%$; $N=5$ ), whereas $\delta^{13} \mathrm{C}_{\text {org }}$ increases and $\delta^{15} \mathrm{~N}_{\text {org }}$ decreases with depth deeper than 500 mbsf. In addition, the downcore profile of $\delta^{13} \mathrm{C}_{\text {org }}$ decreases in the intervals from seafloor to $75 \mathrm{mbsf}$ and from $450 \mathrm{mbsf}$ to the bottom of the hole and is relatively constant between 75 and $450 \mathrm{mbsf}$ at Site U1413. $\delta^{15} \mathrm{~N}_{\text {org }}$ decreases from 150 to $225 \mathrm{mbsf}$ and then gradually increases with depth at Site U1413 (Fig. F3). The variation of $\delta^{13} \mathrm{C}_{\text {org }}$ and $\delta^{15} \mathrm{~N}_{\text {org }}$ at Sites U1380 and U1413 do not correlate with lithostratigraphic units (Figs. F1, F3).

Organic matter produced by land plants through the $\mathrm{C}_{3}$ pathway has an average $\delta^{13} \mathrm{C}_{\text {org }}$ of approximately $-27 \%$ (range $=-32 \%$ to $-21 \%$ VPDB) (Deines, 1980 ); through the $\mathrm{C}_{4}$ pathway, average $\delta^{13} \mathrm{C}_{\text {org }}$ is approximately $-14 \%$ (range $=-17 \%$ o to $-9 \%$ VPDB) (Deines, 1980). Marine organic matter typically has $\delta^{13} \mathrm{C}$ values from $-22 \%$ to $-20 \%$ (Jasper and Gagosian, 1990; Meyers, 1994). Measured $\delta^{13} \mathrm{C}_{\text {org }}$ values do not clearly identify the source of organic matter, rather they reveal that the organic matter in the sediment could be derived from an admixture of marine algae and $\mathrm{C}_{3}$ land plants (Table T1; Figs. F1, F2, F3, and F4). However, the relationship between TOC/TN ratios and $\delta^{13} \mathrm{C}_{\text {org }}$ values leads to a different interpretation. As shown in Figure F6, most organic matter 
at Sites U1380 and U1413 lies in the marine/fresh algal admixture origin, whereas organic matter at Site U1412 predominantly originated from marine algae. There is no correlation of organic matter source with lithostratigraphic units.

\section{Acknowledgments}

This study used samples provided by the Integrated Ocean Drilling Program (IODP) and was financially supported by the Korea Integrated Ocean Drilling Program (K-IODP) of Ministry of Oceans and Fisheries (MOF). We are grateful to Marta E. Torres for constructive comments and improving the manuscript.

\section{References}

Arthur, M.A., Dean, W.E., and Laarkamp, K., 1998. Organic carbon accumulation and preservation in surface sediments on the Peru margin. Chemical Geology, 152(34):273-286. http://dx.doi.org/10.1016/S0009-2541(98)00120-X

Deines, P., 1980. The isotopic composition of reduced organic carbon. In Fritz, P., and Fontes, J.C. (Eds.), Handbook of Environmental Isotope Geochemistry (Volume 1): The Terrestrial Environment, A: Amsterdam (Elsevier), 329-406. http://dx.doi.org/10.1016/B978-0-44441780-0.50015-8

Emerson, S., and Hedges, J.I., 1988. Processes controlling the organic carbon content of open ocean sediments. Paleoceanography, 3(5):621-634. http://dx.doi.org/10.1029/PA003i005p00621

Harris, R.N., Sakaguchi, A., Petronotis, K., Baxter, A.T., Berg, R., Burkett, A., Charpentier, D., Choi, J., Diz Ferreiro, P., Hamahashi, M., Hashimoto, Y., Heydolph, K., Jovane, L., Kastner, M., Kurz, W., Kutterolf, S.O., Li, Y., Malinverno, A., Martin, K.M., Millan, C., Nascimento, D.B., Saito, S., Sandoval Gutierrez, M.I., Screaton, E.J., Smith-Duque, C.E., Solomon, E.A., Straub, S.M., Tanikawa, W., Torres, M.E., Uchimura, H., Vannucchi, P., Yamamoto, Y., Yan, Q., and Zhao, X., 2013. Expedition 344 summary. In Harris, R.N., Sakaguchi, A., Petronotis, $\mathrm{K}$., and the Expedition 344 Scientists, Proceedings of the Integrated Ocean Drilling Program, 344: College Station, TX (Integrated Ocean Drilling Program). http://dx.doi.org/10.2204/iodp.proc.344.101.2013

Jasper, J.P., and Gagosian, R.B., 1990. The sources and deposition of organic matter in the late Quaternary
Pigmy Basin, Gulf of Mexico. Geochimica et Cosmochimica Acta, 54(4):1117-1132.

http://dx.doi.org/10.1016/0016-7037(90)90443-O

Kim, J.-H., Kong, G.-S., Ryu, J.-S., and Park, M.-H., 2014. Revisiting the origin of organic matter and depositional environment of sediment in the central Ulleung Basin, East Sea since the late Quaternary. Quaternary International, 344:181-191. http://dx.doi.org/10.1016/j.quaint.2014.07.022

Kim, J.-H., Park, M.-H., Tsunogai, U., Cheong, T.-J., Ryu, B.J., Lee, Y-.J., Han, H.-C., Oh, J.-H., and Chang, H.-W., 2007. Geochemical characterization of the organic matter, pore water constituents and shallow methane gas in the eastern part of the Ulleung Basin, East Sea (Japan Sea). Island Arc, 16(1):93-104. http://dx.doi.org/ 10.1111/j.1440-1738.2007.00560.x

Lafargue, E., Espitalié, J., Marquis, F., and Pillot, D., 1998. Rock-Eval 6 applications in hydrocarbon exploration, production, and soil contamination studies. Revue de l'Institut Français du Pétrole, 53(4):421-437 (in French). http://dx.doi.org/10.2516/ogst:1998036

Lamb, A.L., Wilson, G.P., and Leng, M.J., 2006. A review of coastal paleoclimate and relative sea-level reconstructions using $\delta^{13} \mathrm{C}$ and $\mathrm{C} / \mathrm{N}$ ratios in organic material. Earth-Science Reviews, 75(1-4):29-57. http://dx.doi.org/10.1016/j.earscirev.2005.10.003

Meyers, P.A., 1994. Preservation of elemental and isotopic source identification of sedimentary organic matter. Chemical Geology, 114(3-4):289-302. http://dx.doi.org/10.1016/0009-2541(94)90059-0

Nali, M., Caccialanza, G., Ghiselli, C., and Chiaramonte, M.A., 2000. $T_{\max }$ of asphaltenes: a parameter for oil maturity assessment. Organic Geochemistry, 31(12):13251332. http://dx.doi.org/10.1016/S01466380(00)00068-1

Peters, K.E., 1986. Guidelines for evaluating petroleum source rock using programmed pyrolysis. AAPG Bulletin, 70(3):318-329. http://archives.datapages.com/data/ bulletns/1986-87/data/pg/0070/0003/0300/ 0318.htm

Tissot, B.P., and Welte, D.H., 1984. Petroleum Formation and Occurrence (2nd edition): Heidelberg (Springer-Verlag).

Initial receipt: 9 August 2015

Acceptance: 12 April 2016

Publication: 15 June 2016

MS 344-204 
Figure F1. Downcore profiles of element and isotopic composition and Rock-Eval pyrolysis, Site U1380. For onboard data, see the Expedition 344 summary chapter (Harris et al., 2013). TC = total carbon, TOC = total organic carbon, TIC = total inorganic carbon, TN = total nitrogen, TS = total sulfur, $T_{\max }=$ maximum $\mathrm{S}_{2}$ temperature, $S_{2}=$ second pyrogram temperature peak, $S_{3}=$ third pyrogram temperature peak, $\mathrm{HI}=$ hydrogen index, OI = oxygen index.

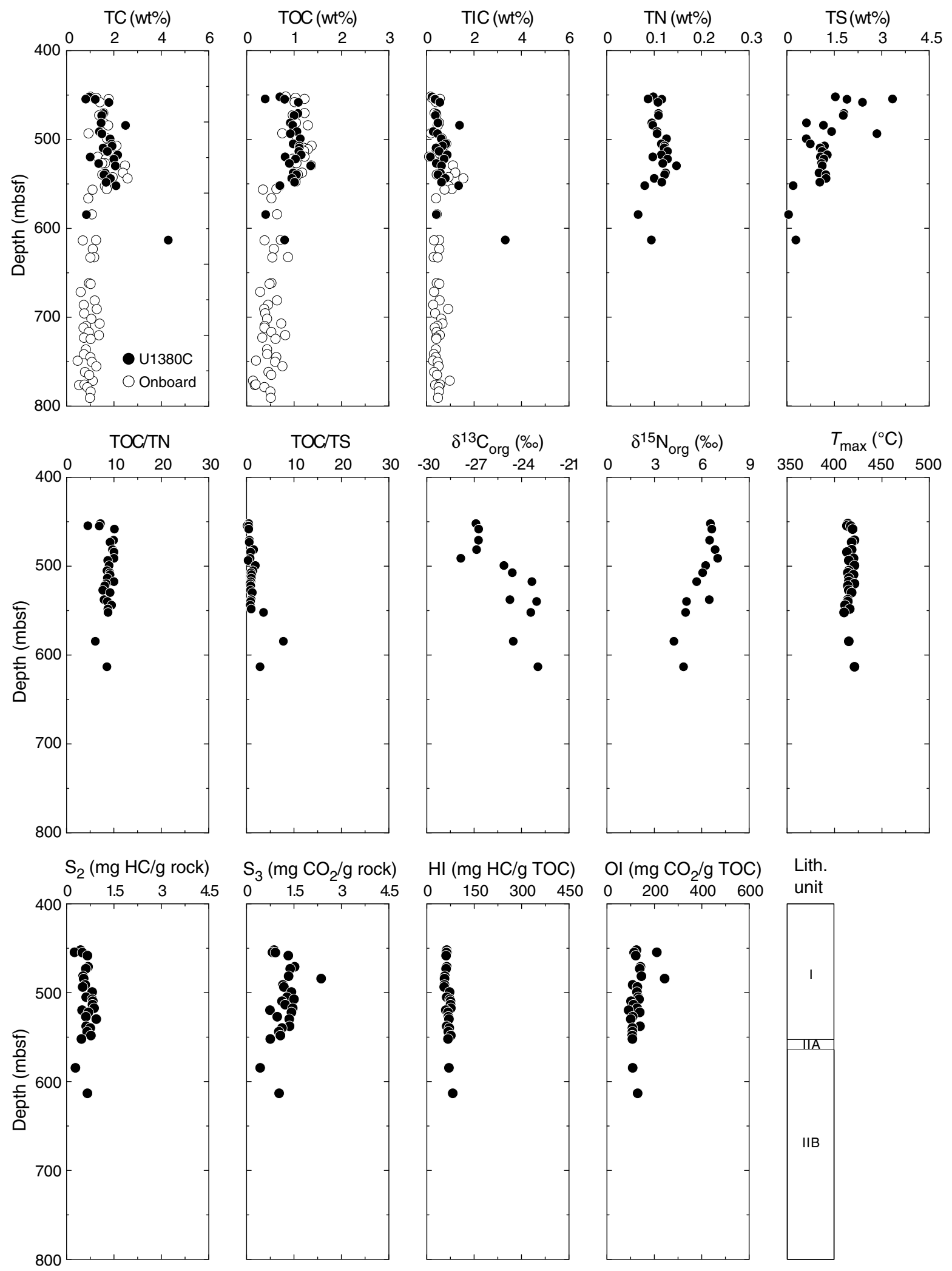


Figure F2. Downcore profiles of element and isotopic composition and Rock-Eval pyrolysis, Site U1412. For onboard data, see the Expedition 344 summary chapter (Harris et al., 2013). TC = total carbon, TOC = total organic carbon, TIC = total inorganic carbon, TN = total nitrogen, TS = total sulfur, $T_{\max }=$ maximum $\mathrm{S}_{2}$ temperature, $S_{2}=$ second pyrogram temperature peak, $S_{3}=$ third pyrogram temperature peak, $\mathrm{HI}=$ hydrogen index, OI = oxygen index.
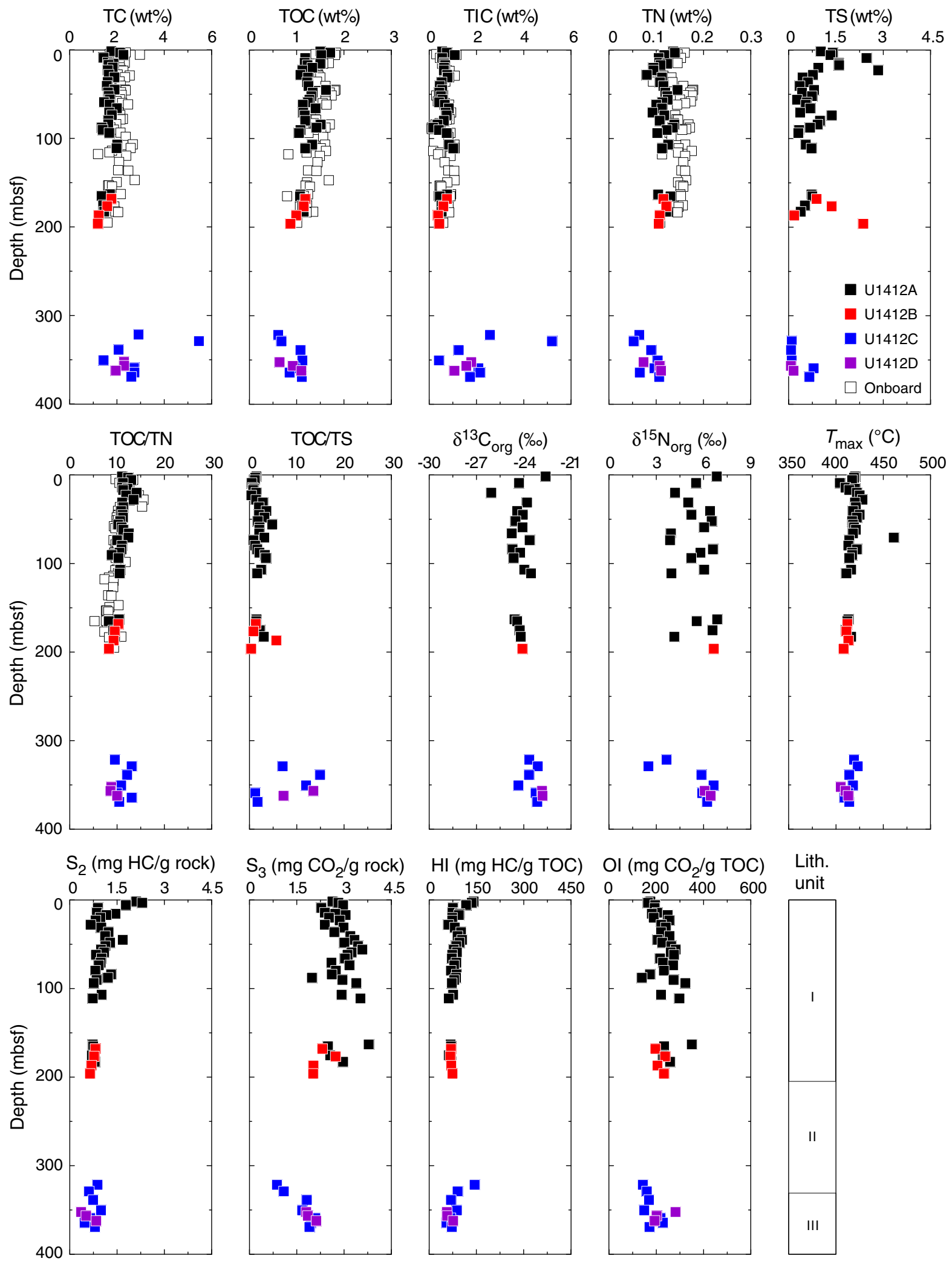

Lith.
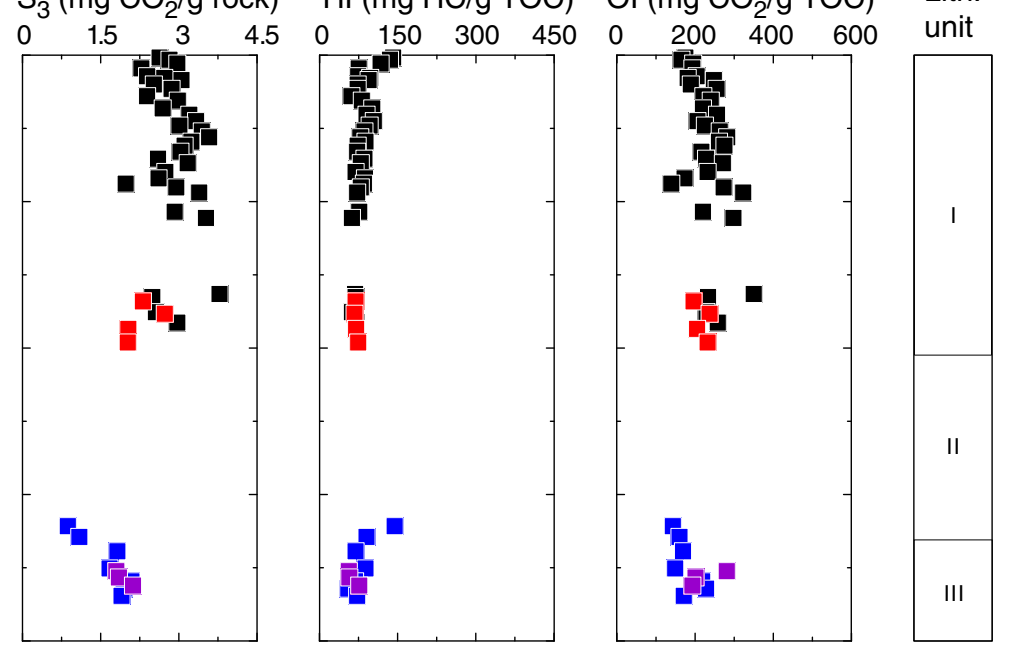
Figure F3. Downcore profiles of element and isotopic composition and Rock-Eval pyrolysis, Site U1413. For onboard data, see the Expedition 344 summary chapter (Harris et al., 2013). TC = total carbon, TOC = total organic carbon, TIC = total inorganic carbon, TN = total nitrogen, TS = total sulfur, $T_{\max }=$ maximum $\mathrm{S}_{2}$ temperature, $S_{2}=$ second pyrogram temperature peak, $S_{3}=$ third pyrogram temperature peak, $\mathrm{HI}=$ hydrogen index, OI = oxygen index.
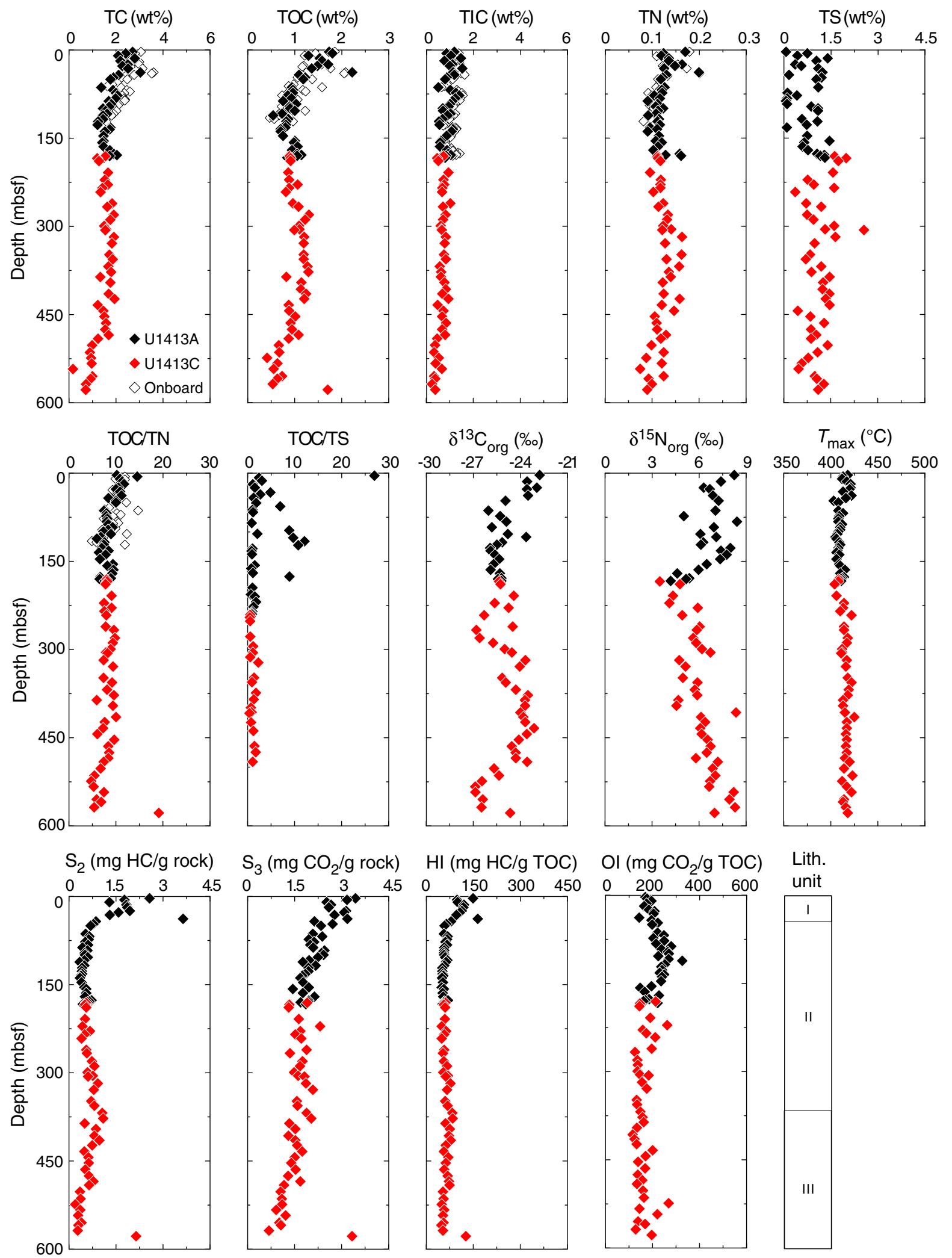

$\mathrm{S}_{3}$ (mg CO$/ \mathrm{g}$ rock)
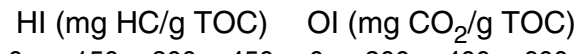

\begin{abstract}
Lith.
\end{abstract}
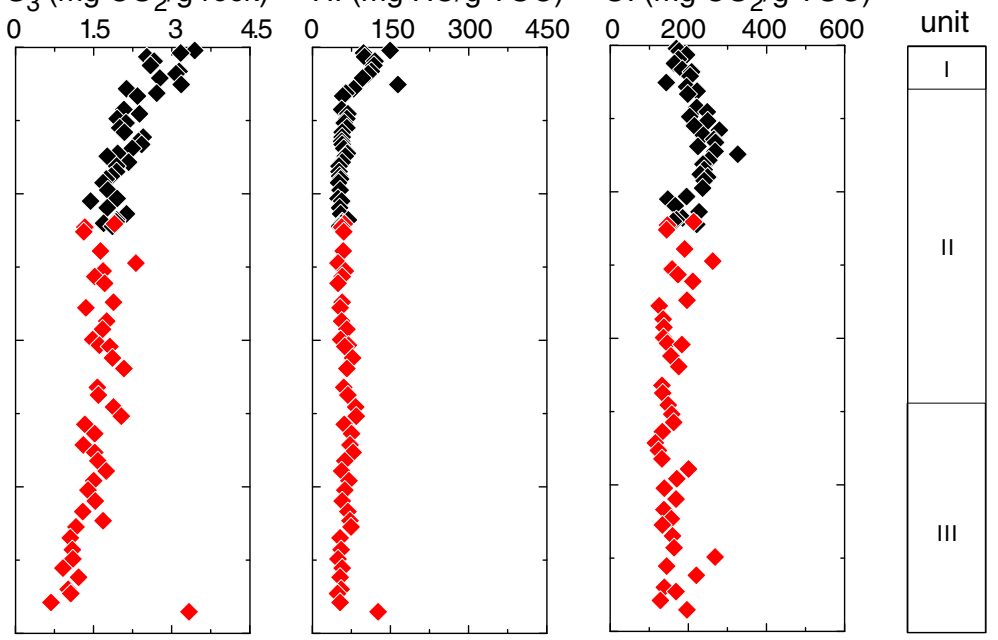
Figure F4. Downcore profiles of element and isotopic composition and Rock-Eval pyrolysis, Site U1414. For onboard data, see the Expedition 344 summary chapter (Harris et al., 2013). TC = total carbon, TOC = total organic carbon, TIC = total inorganic carbon, TN = total nitrogen, TS = total sulfur, $T_{\max }=$ maximum $\mathrm{S}_{2}$ temperature, $S_{2}=$ second pyrogram temperature peak, $S_{3}=$ third pyrogram temperature peak, $\mathrm{HI}=$ hydrogen index, OI = oxygen index.
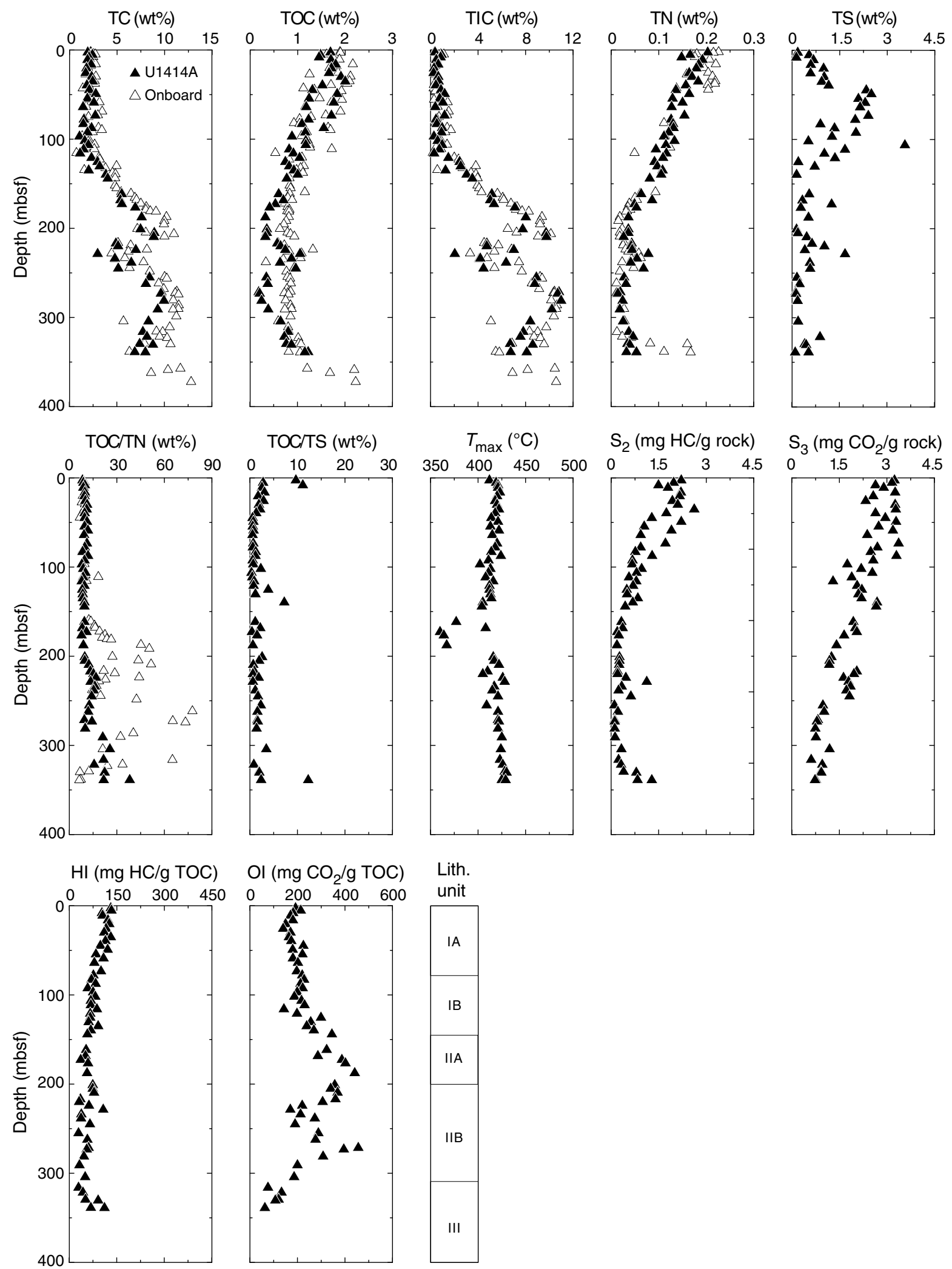

Lith.

unit

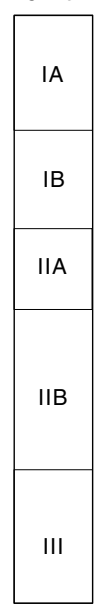


Figure F5. Modified van Krevelen-type diagrams of hydrogen index (HI) versus oxygen index (OI) and crossplots of second pyrogram temperature peak $\left(\mathrm{S}_{2}\right)$ and total organic carbon (TOC). Type I = lacustrine algae, Type II = marine algae, Type III = vascular plants. A. Site U1380. B. Site U1412. C. Site U1413. (Continued on next page.)
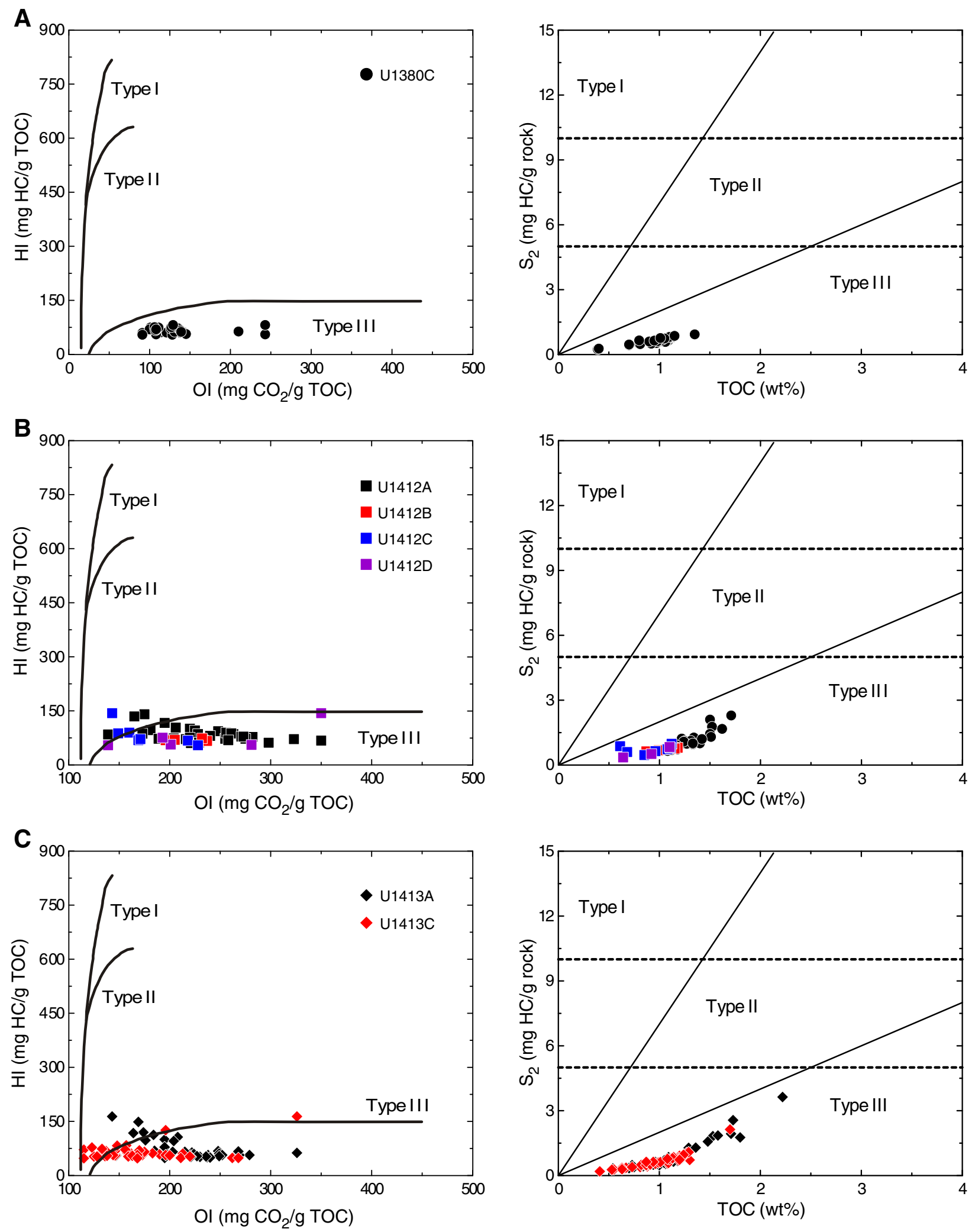
Figure F5 (continued). D. Site U1414.
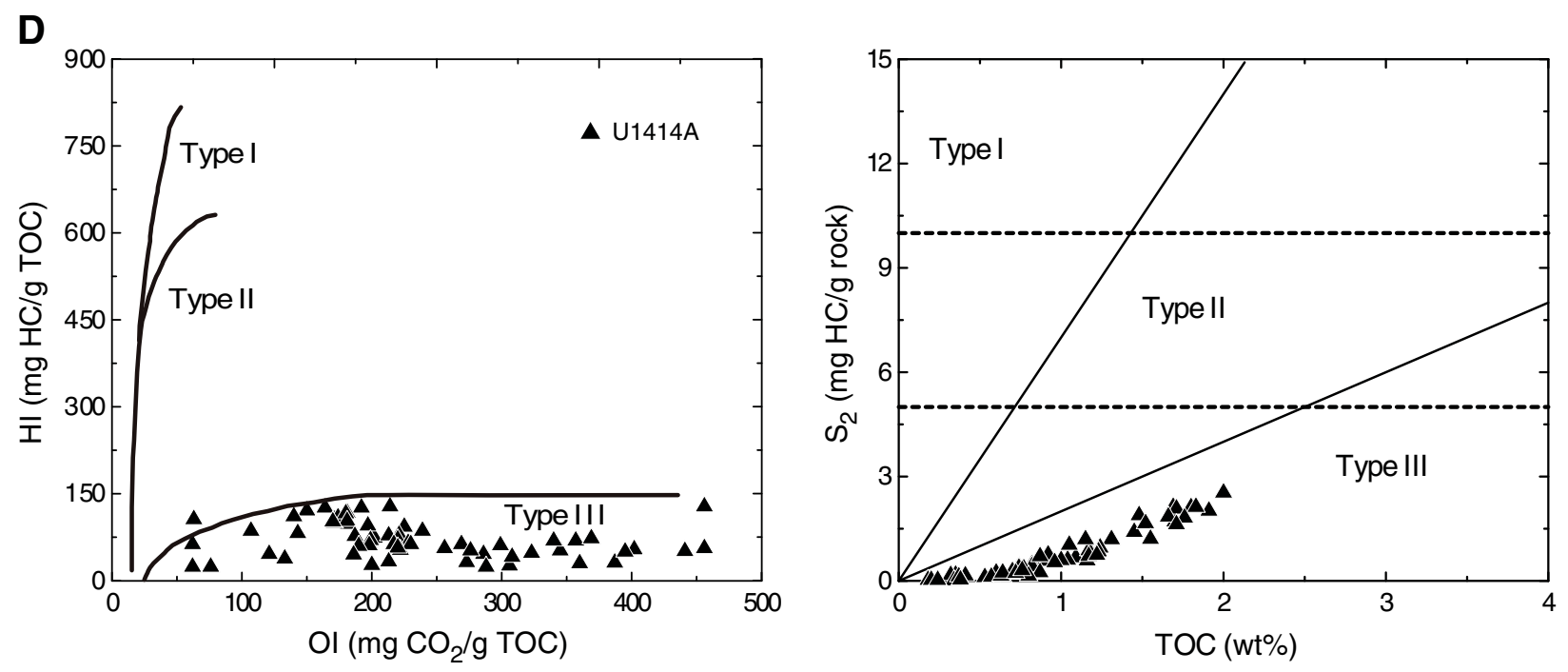
Figure F6. Crossplots of $\delta^{13} \mathrm{C}_{\text {org }}$ and total organic carbon/total nitrogen (TOC/TN) ratios, Sites U1380, U1412, and U1413 (adapted after Lamb et al., 2006).
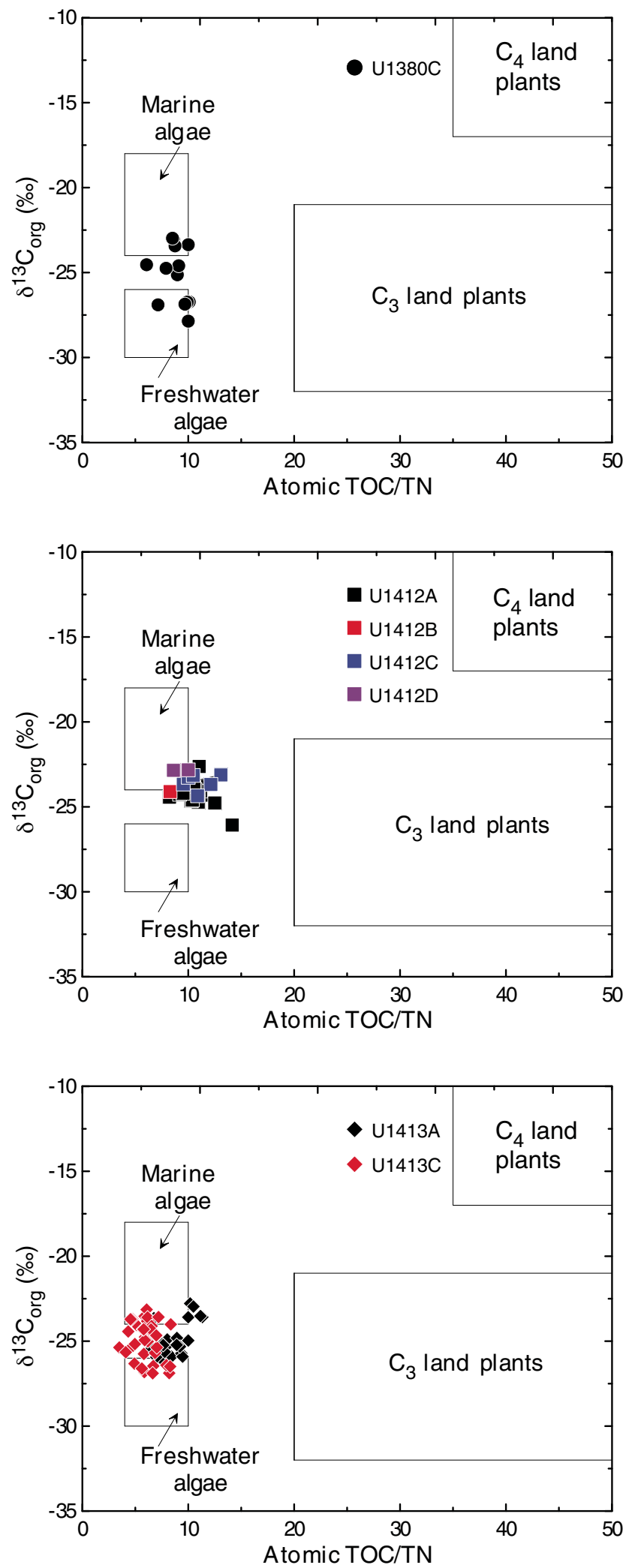
Table T1. Elemental and isotopic compositions and Rock-Eval pyrolysis of bulk sediment, Sites U1380, U1412, U1413, and U1414. (Continued on next four pages).

\begin{tabular}{|c|c|c|c|c|c|c|c|c|c|c|c|c|c|c|c|c|}
\hline $\begin{array}{l}\text { Core, section, } \\
\text { interval }(\mathrm{cm})\end{array}$ & $\begin{array}{l}\text { Depth } \\
\text { (mbsf) }\end{array}$ & $\begin{array}{c}\text { TC } \\
\text { (wt\%) }\end{array}$ & $\begin{array}{l}\text { TOC } \\
\text { (wt\%) }\end{array}$ & $\begin{array}{c}\text { TIC } \\
\text { (wt\%) }\end{array}$ & $\begin{array}{c}\text { TN } \\
\text { (wt\%) }\end{array}$ & $\begin{array}{c}\text { TS } \\
\text { (wt\%) }\end{array}$ & $\mathrm{TOC} / \mathrm{TN}$ & TOC/TS & $\begin{array}{l}\delta^{13} \mathrm{C}_{\mathrm{org}} \\
(\% 0)\end{array}$ & $\begin{array}{l}\delta^{15} \mathrm{~N}_{\mathrm{org}} \\
(\% 0)\end{array}$ & $\begin{array}{c}\mathrm{S}_{1} \\
\text { (mg HC/g rock) }\end{array}$ & $\begin{array}{c}\mathrm{S}_{2} \\
\text { (mg HC/g rock) }\end{array}$ & $\begin{array}{c}\mathrm{S}_{3} \\
\text { (mg CO} \\
2 / g \text { rock) }\end{array}$ & $\begin{array}{l}T_{\max } \\
\left({ }^{\circ} \mathrm{C}\right)\end{array}$ & $\begin{array}{c}\mathrm{HI} \\
\text { (mg HC/g TOC) }\end{array}$ & $\begin{array}{c}\mathrm{Ol} \\
\left(\mathrm{mg} \mathrm{CO}_{2} / \mathrm{g} \text { TOC) }\right.\end{array}$ \\
\hline \multicolumn{17}{|l|}{ 344-U1380C- } \\
\hline $3 R-3,114-144$ & 451.9 & 0.96 & 0.70 & 0.23 & 0.10 & 1.53 & 7.14 & 0.46 & -26.89 & 6.54 & 0.04 & 0.44 & 0.87 & 414 & 63 & 124 \\
\hline $3 R-5,112-142$ & 454.7 & 0.81 & 0.39 & 0.33 & 0.09 & 3.34 & 4.48 & 0.12 & - & - & 0.03 & 0.25 & 0.82 & 413 & 64 & 210 \\
\hline $3 R-5,112-150$ & 454.7 & 1.22 & 0.80 & 0.36 & 0.12 & 1.90 & 6.90 & 0.42 & - & - & 0.05 & 0.50 & 0.91 & 417 & 62 & 114 \\
\hline $4 \mathrm{R}-1,51-73$ & 458.1 & 1.79 & 1.09 & 0.56 & 0.11 & 2.39 & 10.09 & 0.46 & -26.72 & 6.63 & 0.05 & 0.66 & 1.32 & 419 & 61 & 121 \\
\hline 5R-3, 107-137 & 470.7 & 1.56 & 1.08 & 0.42 & 0.11 & 1.80 & 9.91 & 0.60 & -26.73 & 6.49 & 0.06 & 0.68 & 1.52 & 421 & 63 & 141 \\
\hline $5 R-5,86-116$ & 472.9 & 1.49 & 1.00 & 0.37 & 0.11 & 1.78 & 9.17 & 0.56 & - & - & 0.05 & 0.61 & 1.38 & 418 & 61 & 138 \\
\hline $6 \mathrm{R}-3,122-152$ & 481.3 & 1.46 & 0.92 & 0.48 & 0.10 & 0.62 & 9.68 & 1.49 & -26.85 & 6.84 & 0.05 & 0.52 & 1.33 & 418 & 57 & 145 \\
\hline $6 \mathrm{R}-5,120-150$ & 484.0 & 2.49 & 0.97 & 1.39 & 0.10 & 1.16 & 10.00 & 0.84 & - & - & 0.04 & 0.54 & 2.36 & 413 & 56 & 243 \\
\hline $7 R-3,120-150$ & 491.0 & 1.39 & 1.06 & 0.28 & 0.11 & 1.41 & 10.00 & 0.75 & -27.85 & 7.00 & 0.05 & 0.59 & 1.15 & 420 & 56 & 108 \\
\hline 7R-5, 87-107 & 493.4 & 1.50 & 0.92 & 0.46 & 0.11 & 2.85 & 8.68 & 0.32 & - & - & 0.05 & 0.51 & 1.18 & 415 & 55 & 128 \\
\hline $8 R-2,116-146$ & 499.1 & 1.84 & 1.13 & 0.62 & 0.13 & 0.61 & 8.97 & 1.85 & -25.12 & 6.24 & 0.05 & 0.81 & 1.42 & 421 & 72 & 126 \\
\hline $8 R-6,107-137$ & 504.9 & 1.91 & 0.98 & 0.81 & 0.12 & 0.74 & 8.52 & 1.33 & - & - & 0.05 & 0.62 & 1.28 & 415 & 63 & 131 \\
\hline $9 \mathrm{R}-1,102-132$ & 507.2 & 1.93 & 1.11 & 0.67 & 0.12 & 1.19 & 9.10 & 0.93 & -24.59 & 6.05 & 0.06 & 0.81 & 1.50 & 414 & 73 & 135 \\
\hline $9 R-3,71-96$ & 509.7 & 1.55 & 1.11 & 0.40 & 0.12 & 1.05 & 9.17 & 1.06 & - & - & 0.06 & 0.83 & 1.12 & 420 & 75 & 101 \\
\hline 9R-6, 79-109 & 513.3 & 1.73 & 1.10 & 0.53 & 0.13 & 1.11 & 8.59 & 0.99 & - & - & 0.06 & 0.82 & 1.22 & 415 & 75 & 111 \\
\hline $10 \mathrm{R}-2,35-65$ & 517.3 & 2.16 & 1.15 & 0.87 & 0.12 & 1.28 & 10.00 & 0.90 & -23.35 & 5.67 & 0.07 & 0.87 & 1.46 & 415 & 76 & 127 \\
\hline $10 R-4,53-78$ & 519.5 & 1.00 & 0.81 & 0.16 & 0.10 & 1.06 & 8.35 & 0.76 & - & - & 0.04 & 0.49 & 0.74 & 421 & 60 & 91 \\
\hline $10 R-6,64-90$ & 521.9 & 2.00 & 1.03 & 0.74 & 0.13 & 1.17 & 8.05 & 0.88 & - & - & 0.06 & 0.69 & 1.42 & 414 & 67 & 138 \\
\hline $11 \mathrm{R}-2,59-89$ & 526.9 & 1.36 & 0.90 & 0.41 & 0.12 & 1.09 & 7.63 & 0.82 & - & - & 0.05 & 0.61 & 0.97 & 415 & 68 & 108 \\
\hline $11 R-4,87-117$ & 529.5 & 2.06 & 1.35 & 0.63 & 0.15 & 1.12 & 9.18 & 1.21 & - & - & 0.07 & 0.94 & 1.35 & 418 & 70 & 100 \\
\hline $12 \mathrm{R}-2,79-113$ & 537.5 & 1.64 & 0.98 & 0.58 & 0.12 & 1.01 & 7.90 & 0.97 & -24.74 & 6.47 & 0.06 & 0.62 & 1.36 & 414 & 63 & 139 \\
\hline $12 \mathrm{R}-4,78-108$ & 539.6 & 1.59 & 1.05 & 0.48 & 0.12 & 1.23 & 8.61 & 0.85 & -23.05 & 5.03 & 0.06 & 0.75 & 1.12 & 414 & 71 & 107 \\
\hline $12 \mathrm{R}-8,62-92$ & 543.7 & 1.86 & 0.95 & 0.79 & 0.10 & 1.24 & 9.50 & 0.77 & - & - & 0.08 & 0.65 & 1.02 & 411 & 68 & 107 \\
\hline $13 R-3,50-80$ & 548.0 & 1.67 & 1.01 & 0.63 & 0.12 & 1.04 & 8.71 & 0.97 & - & - & 0.06 & 0.77 & 1.07 & 416 & 76 & 106 \\
\hline $13 R-6,58-88$ & 551.9 & 2.09 & 0.70 & 1.35 & 0.08 & 0.20 & 8.75 & 3.58 & -23.42 & 4.97 & 0.04 & 0.47 & 0.75 & 410 & 67 & 107 \\
\hline $17 \mathrm{R}-1,63-90$ & 584.4 & 0.84 & 0.40 & 0.41 & 0.07 & 0.05 & 6.06 & 7.76 & -24.53 & 4.23 & 0.03 & 0.28 & 0.43 & 415 & 70 & 108 \\
\hline $20 \mathrm{R}-1,35-65$ & 613.4 & 4.30 & 0.80 & 3.32 & 0.09 & 0.28 & 8.51 & 2.84 & -22.97 & 4.84 & 0.04 & 0.66 & 1.03 & 421 & 82 & 129 \\
\hline \multicolumn{17}{|l|}{ 344-U1412A- } \\
\hline $1 \mathrm{H}-1,138-150$ & 1.4 & 1.77 & 1.50 & 0.55 & 0.14 & 1.02 & 11.03 & 1.47 & -22.61 & 6.84 & 0.24 & 2.11 & 2.63 & 420 & 141 & 175 \\
\hline $1 \mathrm{H}-2,138-150$ & 2.9 & 2.13 & 1.71 & 0.74 & 0.14 & 1.39 & 12.30 & 1.23 & - & - & 0.23 & 2.30 & 2.82 & 419 & 135 & 165 \\
\hline $1 \mathrm{H}-4,98-110$ & 5.5 & 2.27 & 1.52 & 1.08 & 0.12 & 1.32 & 12.99 & 1.15 & - & - & 0.19 & 1.78 & 2.97 & 417 & 117 & 195 \\
\hline $2 \mathrm{H}-2,138-150$ & 8.8 & 1.44 & 1.18 & 0.55 & 0.11 & 2.47 & 11.24 & 0.48 & -24.30 & 5.52 & 0.14 & 0.89 & 2.28 & 404 & 75 & 193 \\
\hline $2 \mathrm{H}-6,72-84$ & 14.2 & 1.61 & 1.17 & 0.64 & 0.11 & 1.59 & 11.04 & 0.74 & - & - & 0.13 & 0.86 & 2.39 & 410 & 74 & 204 \\
\hline $2 \mathrm{H}-7,106-118$ & 15.4 & 1.85 & 1.50 & 0.63 & 0.12 & 1.59 & 12.20 & 0.94 & - & - & 0.18 & 1.45 & 2.72 & 420 & 97 & 181 \\
\hline $3 \mathrm{H}-1,138-150$ & 16.8 & 1.64 & 1.23 & 0.67 & 0.11 & 1.60 & 11.28 & 0.77 & - & - & 0.14 & 1.16 & 3.05 & 414 & 94 & 248 \\
\hline $3 \mathrm{H}-3,138-150$ & 19.8 & 1.82 & 1.33 & 0.63 & 0.09 & 0.94 & 14.15 & 1.42 & -26.06 & 4.19 & 0.12 & 0.97 & 2.52 & 422 & 73 & 189 \\
\hline $3 \mathrm{H}-5,138-150$ & 22.8 & 1.61 & 1.12 & 0.62 & 0.09 & 2.84 & 11.91 & 0.39 & - & - & 0.10 & 0.82 & 2.86 & 425 & 73 & 255 \\
\hline $4 \mathrm{H}-2,138-150$ & 27.8 & 1.65 & 1.08 & 0.76 & 0.08 & 0.77 & 13.50 & 1.40 & - & - & 0.09 & 0.66 & 2.39 & 428 & 61 & 221 \\
\hline $4 \mathrm{H}-4,138-150$ & 30.9 & 1.90 & 1.24 & 0.78 & 0.11 & 0.44 & 11.07 & 2.82 & -23.79 & 5.02 & 0.13 & 1.00 & 2.98 & 420 & 81 & 240 \\
\hline $5 \mathrm{H}-3,118-135$ & 36.2 & 1.61 & 1.22 & 0.53 & 0.11 & 0.61 & 11.30 & 2.00 & - & - & 0.15 & 1.23 & 2.69 & 421 & 101 & 220 \\
\hline $5 \mathrm{H}-7,133-150$ & 40.5 & 1.57 & 1.25 & 0.45 & 0.12 & 0.35 & 10.87 & 3.58 & -24.42 & 6.41 & 0.14 & 1.12 & 3.20 & 418 & 90 & 256 \\
\hline $6 \mathrm{H}-2,132-149$ & 44.9 & 1.90 & 1.62 & 0.55 & 0.15 & 0.80 & 11.17 & 2.02 & -24.06 & 5.23 & 0.17 & 1.68 & 3.33 & 425 & 104 & 206 \\
\hline $6 \mathrm{H}-4,134-151$ & 47.9 & 1.62 & 1.34 & 0.43 & 0.12 & 0.42 & 11.26 & 3.17 & - & - & 0.15 & 1.28 & 3.01 & 422 & 96 & 225 \\
\hline 7H-2, 128-145 & 51.9 & 1.65 & 1.31 & 0.50 & 0.12 & 0.74 & 10.65 & 1.78 & -24.52 & 6.51 & 0.15 & 1.13 & 3.44 & 417 & 86 & 263 \\
\hline 7H-5, 103-120 & 55.9 & 1.70 & 1.27 & 0.56 & 0.12 & 0.26 & 10.24 & 4.82 & - & - & 0.14 & 0.99 & 3.58 & 418 & 78 & 282 \\
\hline $8 \mathrm{H}-1,129-151$ & 58.9 & 1.45 & 1.24 & 0.42 & 0.11 & 0.58 & 10.97 & 2.15 & -24.08 & 6.04 & 0.18 & 1.09 & 3.24 & 419 & 88 & 261 \\
\hline $8 \mathrm{H}-3,100-122$ & 61.6 & 1.69 & 1.13 & 0.66 & 0.10 & 0.54 & 11.30 & 2.08 & - & - & 0.14 & 0.83 & 3.11 & 421 & 73 & 275 \\
\hline $9 \mathrm{H}-2,128-150$ & 65.8 & 2.00 & 1.40 & 0.83 & 0.11 & 0.68 & 12.50 & 2.05 & -24.76 & 3.93 & 0.19 & 1.01 & 3.03 & 418 & 72 & 216 \\
\hline
\end{tabular}


Table T1 (continued). (Continued on next page).

\begin{tabular}{|c|c|c|c|c|c|c|c|c|c|c|c|c|c|c|c|c|}
\hline $\begin{array}{l}\text { Core, section, } \\
\text { interval }(\mathrm{cm})\end{array}$ & $\begin{array}{l}\text { Depth } \\
\text { (mbsf) }\end{array}$ & $\begin{array}{c}\text { TC } \\
\text { (wt\%) }\end{array}$ & $\begin{array}{l}\text { TOC } \\
\text { (wt\%) }\end{array}$ & $\begin{array}{c}\text { TIC } \\
\text { (wt\%) }\end{array}$ & $\begin{array}{c}\mathrm{TN} \\
(\mathrm{wt} \%)\end{array}$ & $\begin{array}{c}\text { TS } \\
\text { (wt\%) }\end{array}$ & TOC/TN & TOC/TS & $\begin{array}{c}\delta^{13} \mathrm{C}_{\text {org }} \\
(\%)\end{array}$ & $\begin{array}{c}\delta^{15} N_{\text {org }} \\
(\% \circ)\end{array}$ & $\begin{array}{c}\mathrm{S}_{1} \\
\text { (mg HC/g rock) }\end{array}$ & $\begin{array}{c}\mathrm{S}_{2} \\
\text { (mg HC/g rock) }\end{array}$ & $\begin{array}{c}\mathrm{S}_{3} \\
\text { (mg CO} \\
2 / \mathrm{g} \text { rock) }\end{array}$ & $\begin{array}{l}T_{\max } \\
\left({ }^{\circ} \mathrm{C}\right)\end{array}$ & $\begin{array}{c}\mathrm{HI} \\
(\mathrm{mg} \mathrm{HC} / \mathrm{g} \text { TOC) }\end{array}$ & $\begin{array}{c}\mathrm{OI} \\
\text { (mg CO} 2 / \mathrm{g} \text { TOC) }\end{array}$ \\
\hline $9 \mathrm{H}-6,135-157$ & 70.5 & 1.67 & 1.14 & 0.70 & 0.09 & 0.36 & 12.39 & 3.13 & - & - & 0.17 & 0.98 & 2.60 & - & 86 & 228 \\
\hline $10 \mathrm{H}-1,128-150$ & 73.6 & 1.79 & 1.17 & 0.74 & 0.12 & 1.37 & 10.00 & 0.86 & -23.63 & 3.87 & 0.17 & 0.92 & 3.17 & 414 & 79 & 271 \\
\hline $10 \mathrm{H}-5,134-156$ & 79.6 & 1.64 & 1.18 & 0.62 & 0.11 & 0.99 & 11.03 & 1.19 & - & - & 0.16 & 0.81 & 2.74 & 413 & 69 & 232 \\
\hline $11 \mathrm{H}-2,130-152$ & 83.9 & 1.63 & 1.51 & 0.35 & 0.14 & 0.92 & 10.94 & 1.65 & -24.71 & 6.59 & 0.19 & 1.31 & 2.61 & 422 & 87 & 173 \\
\hline $12 \mathrm{H}-1,115-137$ & 87.6 & 1.36 & 1.42 & 0.17 & 0.14 & 0.68 & 10.52 & 2.09 & -24.23 & 5.80 & 0.20 & 1.21 & 1.98 & 417 & 85 & 139 \\
\hline $12 \mathrm{H}-3,94-116$ & 90.1 & 1.40 & 1.08 & 0.41 & 0.12 & 0.33 & 8.85 & 3.26 & - & - & 0.14 & 0.85 & 2.95 & 417 & 79 & 273 \\
\hline $13 \mathrm{H}-2,124-146$ & 93.6 & 1.67 & 1.05 & 0.74 & 0.10 & 0.30 & 10.29 & 3.46 & -24.64 & 5.22 & 0.13 & 0.76 & 3.39 & 414 & 72 & 323 \\
\hline $15 \mathrm{H}-2,93-115$ & 106.8 & 2.01 & 1.33 & 0.84 & 0.13 & 0.54 & 10.64 & 2.46 & -23.97 & 6.04 & 0.19 & 1.01 & 2.92 & 416 & 76 & 220 \\
\hline $16 \mathrm{X}-2,126-148$ & 111.0 & 1.98 & 1.18 & 1.02 & 0.11 & 0.72 & 10.54 & 1.64 & -23.54 & 3.96 & 0.16 & 0.73 & 3.52 & 411 & 62 & 298 \\
\hline $22 X-1,131-151$ & 162.9 & 1.70 & 1.08 & 0.76 & 0.10 & 0.72 & 10.38 & 1.50 & -24.59 & 6.86 & 0.16 & 0.73 & 3.78 & 413 & 68 & 350 \\
\hline $22 X-3,68-88$ & 165.0 & 1.35 & 1.07 & 0.45 & 0.13 & 0.75 & 8.23 & - & -24.42 & 5.56 & 0.10 & 0.74 & 2.49 & 412 & 69 & 233 \\
\hline $23 X-3,100-120$ & 175.3 & 1.42 & 1.12 & 0.53 & 0.12 & 0.51 & 9.18 & 2.19 & -24.25 & 6.57 & 0.10 & 0.70 & 2.56 & 410 & 62 & 229 \\
\hline $24 X-2,56-81$ & 182.5 & 1.46 & 1.15 & 0.53 & 0.12 & 0.38 & 9.50 & 3.04 & -24.19 & 4.14 & 0.14 & 0.79 & 2.97 & 416 & 69 & 258 \\
\hline \multicolumn{17}{|l|}{ 344-U1412B- } \\
\hline $4 X-2,100-120$ & 168.0 & 1.77 & 1.18 & 0.75 & 0.12 & 0.88 & 10.26 & 1.34 & - & - & 0.12 & 0.81 & 2.31 & 412 & 69 & 196 \\
\hline $5 X-1,120-140$ & 176.4 & 1.59 & 1.15 & 0.60 & 0.12 & 1.36 & 9.50 & 0.84 & - & - & 0.10 & 0.77 & 2.73 & 411 & 67 & 237 \\
\hline $6 \mathrm{X}-2,99-125$ & 186.7 & 1.23 & 0.99 & 0.38 & 0.11 & 0.17 & 9.25 & 5.67 & - & - & 0.10 & 0.69 & 2.03 & 413 & 70 & 205 \\
\hline $7 X-2,51-73$ & 196.0 & 1.19 & 0.87 & 0.43 & 0.11 & 2.37 & 8.29 & 0.37 & -24.09 & 6.65 & 0.09 & 0.64 & 2.02 & 408 & 74 & 232 \\
\hline \multicolumn{17}{|l|}{ 344-U1412C- } \\
\hline 4R-CC, 19-24 & 321.5 & 2.91 & 0.61 & 2.57 & 0.06 & 0.03 & 9.53 & 19.75 & -23.65 & 3.65 & 0.07 & 0.88 & 0.87 & 419 & 144 & 143 \\
\hline $5 R-1,0-36$ & 328.8 & 5.47 & 0.68 & 5.19 & 0.05 & 0.10 & 13.08 & 7.02 & -23.11 & 2.50 & 0.03 & 0.61 & 1.09 & 423 & 90 & 160 \\
\hline $6 \mathrm{R}-1,9-20$ & 338.6 & 2.07 & 1.08 & 1.25 & 0.09 & 0.07 & 12.13 & 14.94 & -23.67 & 5.86 & 0.08 & 0.74 & 1.82 & 414 & 69 & 169 \\
\hline $7 \mathrm{R}-2,96-112$ & 350.3 & 1.44 & 1.12 & 0.41 & 0.10 & 0.09 & 10.87 & 11.97 & -24.34 & 6.63 & 0.10 & 0.99 & 1.67 & 418 & 88 & 149 \\
\hline $8 \mathrm{R}-1,121-148$ & 359.1 & 2.72 & 0.96 & 2.08 & 0.10 & 0.79 & 10.00 & - & -23.25 & 5.91 & 0.08 & 0.65 & 2.09 & 413 & 68 & 218 \\
\hline $8 R-5,59-95$ & 364.1 & 2.74 & 0.85 & 2.15 & 0.07 & - & 13.08 & - & - & - & 0.07 & 0.47 & 1.94 & 409 & 55 & 228 \\
\hline $9 R-2,58-88$ & 369.0 & 2.61 & 1.11 & 1.73 & 0.11 & 0.66 & 10.47 & 1.69 & -23.14 & 6.23 & 0.07 & 0.80 & 1.90 & 414 & 72 & 171 \\
\hline \multicolumn{17}{|l|}{ 344-U1412D- } \\
\hline 2R-2, 78-101 & 352.2 & 2.30 & 0.64 & 1.78 & 0.07 & 0.01 & 8.77 & 72.26 & - & - & 0.07 & 0.36 & 1.80 & 405 & 56 & 281 \\
\hline $2 \mathrm{R}-5,106-131$ & 356.5 & 2.31 & 0.92 & 1.57 & 0.11 & 0.07 & 8.60 & 13.51 & -22.84 & 6.07 & 0.07 & 0.52 & 1.85 & 410 & 57 & 201 \\
\hline $3 R-3,61-86$ & 362.0 & 1.95 & 1.10 & 1.07 & 0.11 & 0.15 & 10.00 & 7.18 & -22.81 & 6.45 & 0.09 & 0.84 & 2.12 & 413 & 76 & 193 \\
\hline \multicolumn{17}{|l|}{ 344-U1413A- } \\
\hline $1 \mathrm{H}-2,138-150$ & 3.0 & 2.71 & 1.73 & 1.18 & 0.17 & 0.06 & 10.18 & 26.96 & -22.77 & 8.24 & 0.30 & 2.57 & 2.92 & 418 & 149 & 169 \\
\hline $1 \mathrm{H}-4,110-122$ & 5.6 & 2.41 & 1.80 & 0.85 & 0.12 & 0.74 & 14.52 & 2.42 & - & - & 0.24 & 1.77 & 3.17 & 413 & 98 & 176 \\
\hline $2 \mathrm{H}-2,138-150$ & 9.5 & 2.09 & 1.29 & 1.04 & 0.12 & 0.43 & 10.84 & 3.03 & - & - & 0.17 & 1.29 & 2.52 & 412 & 100 & 195 \\
\hline $2 \mathrm{H}-5,138-150$ & 14.0 & 2.80 & 1.53 & 1.46 & 0.14 & 1.39 & 11.33 & 1.10 & -23.57 & 7.39 & 0.20 & 1.83 & 2.66 & 417 & 120 & 174 \\
\hline $3 \mathrm{H}-2,100-112$ & 18.3 & 2.19 & 1.58 & 0.76 & 0.14 & 1.04 & 11.70 & 1.53 & - & - & 0.22 & 1.86 & 2.59 & 421 & 118 & 164 \\
\hline $3 \mathrm{H}-6,140-152$ & 24.4 & 2.50 & 1.71 & 1.10 & 0.16 & 0.35 & 10.49 & 4.83 & -22.95 & 6.29 & 0.20 & 1.94 & 3.14 & 421 & 113 & 184 \\
\hline $4 \mathrm{H}-1,136-151$ & 27.0 & 2.27 & 1.48 & 0.99 & 0.15 & 0.55 & 10.00 & 2.69 & -23.58 & 6.68 & 0.20 & 1.58 & 3.08 & 420 & 107 & 208 \\
\hline $4 \mathrm{H}-4,137-152$ & 31.3 & 2.51 & 1.36 & 1.52 & 0.13 & 1.09 & 10.88 & 1.24 & - & - & 0.18 & 1.30 & 2.77 & 413 & 96 & 204 \\
\hline $5 \mathrm{H}-2,135-150$ & 37.9 & 3.04 & 2.22 & 1.13 & 0.20 & 1.23 & 11.16 & 1.81 & -23.51 & 6.88 & 0.30 & 3.64 & 3.18 & 422 & 164 & 143 \\
\hline $5 \mathrm{H}-5,135-150$ & 42.2 & 2.11 & 1.09 & 1.18 & 0.13 & 0.16 & 8.38 & 6.93 & - & - & 0.12 & 0.86 & 2.13 & 416 & 79 & 195 \\
\hline $6 \mathrm{H}-2,137-152$ & 46.7 & 1.89 & 1.22 & 0.86 & 0.12 & 1.20 & 10.00 & 1.02 & -24.95 & 7.24 & 0.15 & 0.78 & 2.71 & 403 & 64 & 222 \\
\hline $6 \mathrm{H}-4,135-150$ & 49.7 & 1.76 & 1.18 & 0.79 & 0.12 & 1.03 & 10.00 & 1.14 & - & - & 0.11 & 0.69 & 2.34 & 408 & 58 & 198 \\
\hline $8 \mathrm{H}-2,131-151$ & 63.4 & 1.37 & 0.94 & 0.50 & 0.13 & 1.09 & 7.46 & 0.86 & -26.04 & 7.04 & 0.10 & 0.53 & 2.08 & 408 & 56 & 221 \\
\hline $8 \mathrm{H}-5,136-156$ & 67.9 & 1.88 & 0.96 & 1.01 & 0.12 & - & 8.00 & - & - & - & 0.10 & 0.65 & 2.38 & 413 & 68 & 248 \\
\hline $9 \mathrm{H}-2,132-154$ & 72.8 & 2.00 & 0.96 & 1.25 & 0.12 & 0.11 & 7.93 & 8.85 & -25.29 & 5.02 & 0.10 & 0.63 & 1.95 & 409 & 66 & 203 \\
\hline $9 \mathrm{H}-5,133-155$ & 77.2 & 2.06 & 0.85 & 1.39 & 0.10 & 0.41 & 8.17 & 2.07 & - & - & 0.09 & 0.52 & 2.12 & 408 & 61 & 249 \\
\hline $10 \mathrm{H}-2,130-152$ & 82.2 & 1.98 & 0.93 & 1.22 & 0.12 & 0.10 & 8.02 & 9.69 & -24.89 & 8.41 & 0.10 & 0.61 & 2.00 & 408 & 66 & 215 \\
\hline $10 \mathrm{H}-5,131-153$ & 86.7 & 1.69 & 0.75 & 1.10 & 0.09 & 0.06 & 8.33 & 12.07 & - & - & 0.07 & 0.43 & 2.09 & 412 & 57 & 279 \\
\hline
\end{tabular}


Table T1 (continued). (Continued on next page).

\begin{tabular}{|c|c|c|c|c|c|c|c|c|c|c|c|c|c|c|c|c|}
\hline $\begin{array}{l}\text { Core, section, } \\
\text { interval }(\mathrm{cm})\end{array}$ & $\begin{array}{l}\text { Depth } \\
\text { (mbsf) }\end{array}$ & $\begin{array}{c}\text { TC } \\
\text { (wt\%) }\end{array}$ & $\begin{array}{c}\text { TOC } \\
\text { (wt\%) }\end{array}$ & $\begin{array}{c}\mathrm{TIC} \\
\text { (wt\%) }\end{array}$ & $\begin{array}{c}\mathrm{TN} \\
(\mathrm{wt} \%)\end{array}$ & $\begin{array}{c}\text { TS } \\
\text { (wt\%) }\end{array}$ & TOC/TN & TOC/TS & $\begin{array}{c}\delta^{13} C_{\text {org }} \\
(\% o)\end{array}$ & $\begin{array}{c}\delta^{15} N_{\text {org }} \\
(\% \circ)\end{array}$ & $\begin{array}{c}\mathrm{S}_{1} \\
\text { (mg HC/g rock) }\end{array}$ & $\begin{array}{c}\mathrm{S}_{2} \\
\text { (mg HC/g rock) }\end{array}$ & $\begin{array}{c}\mathrm{S}_{3} \\
\text { (mg CO} \\
2 / \mathrm{g} \text { rock) }\end{array}$ & $\begin{array}{l}T_{\max } \\
\left({ }^{\circ} \mathrm{C}\right)\end{array}$ & $\begin{array}{c}\mathrm{HI} \\
(\mathrm{mg} \mathrm{HC} / \mathrm{g} \text { TOC) }\end{array}$ & $\begin{array}{c}\mathrm{OI} \\
\text { (mg CO} 2 / \mathrm{g} \text { TOC) }\end{array}$ \\
\hline $11 \mathrm{H}-2,129-151$ & 91.7 & 1.80 & 1.03 & 1.00 & 0.11 & 0.10 & 9.28 & 10.76 & -25.82 & 6.94 & 0.10 & 0.59 & 2.45 & 410 & 57 & 238 \\
\hline $11 \mathrm{H}-5,130-152$ & 96.1 & 1.71 & 0.89 & 0.94 & 0.11 & 0.87 & 8.32 & 1.03 & - & - & 0.08 & 0.50 & 2.36 & 410 & 56 & 265 \\
\hline $12 \mathrm{H}-1,128-150$ & 99.2 & 1.45 & 0.90 & 0.69 & 0.12 & 1.08 & 7.32 & 0.83 & - & - & 0.11 & 0.51 & 2.42 & 408 & 57 & 269 \\
\hline $12 \mathrm{H}-4,109-131$ & 103.3 & 1.56 & 1.00 & 0.70 & 0.11 & 1.08 & 8.93 & 0.92 & -24.80 & 6.08 & 0.10 & 0.60 & 2.24 & 407 & 60 & 224 \\
\hline $13 \mathrm{H}-2,130-152$ & 108.3 & 1.62 & 0.73 & 1.02 & 0.11 & - & 6.76 & - & -23.63 & 7.09 & 0.08 & 0.49 & 1.96 & 405 & 67 & 268 \\
\hline $13 \mathrm{H}-4,130-152$ & 111.3 & 1.45 & 0.54 & 0.89 & 0.09 & - & 5.93 & - & - & - & 0.06 & 0.34 & 1.76 & 406 & 63 & 326 \\
\hline $14 \mathrm{H}-3,128-150$ & 117.4 & 1.45 & 0.86 & 0.68 & 0.11 & 0.57 & 7.54 & 1.50 & -25.16 & 6.26 & 0.09 & 0.49 & 2.17 & 409 & 57 & 252 \\
\hline $15 \mathrm{H}-2,130-152$ & 121.4 & 1.24 & 0.82 & 0.53 & 0.11 & 1.07 & 7.39 & 0.77 & -25.50 & 6.11 & 0.08 & 0.42 & 1.94 & 410 & 51 & 237 \\
\hline $16 \mathrm{H}-2,126-150$ & 127.1 & 1.22 & 0.81 & 0.55 & 0.12 & 0.72 & 6.98 & 1.12 & -25.93 & 7.99 & 0.07 & 0.41 & 1.94 & 408 & 51 & 240 \\
\hline $17 \mathrm{H}-2,80-102$ & 131.7 & 1.77 & 0.80 & 1.10 & 0.10 & 0.09 & 8.42 & 8.89 & -25.90 & 7.39 & 0.08 & 0.42 & 1.84 & 407 & 52 & 230 \\
\hline $17 \mathrm{H}-4,128-150$ & 134.6 & 1.73 & 0.70 & 1.12 & 0.11 & 0.06 & 6.36 & 11.14 & - & - & 0.07 & 0.38 & 1.74 & 406 & 54 & 249 \\
\hline $18 \mathrm{H}-2,130-152$ & 138.4 & 1.53 & 0.70 & 1.06 & 0.09 & 0.04 & 7.78 & 19.73 & -25.62 & 7.75 & 0.06 & 0.35 & 1.68 & 409 & 50 & 240 \\
\hline $19 X-4,97-122$ & 145.9 & 1.47 & 0.75 & 0.85 & 0.11 & 0.74 & 6.58 & - & -25.35 & 7.34 & 0.07 & 0.40 & 1.77 & 406 & 53 & 236 \\
\hline $20 X-4,65-90$ & 154.6 & 1.47 & 1.00 & 0.61 & 0.11 & 1.45 & 9.35 & 0.69 & -25.71 & 6.48 & 0.10 & 0.49 & 1.95 & 409 & 49 & 195 \\
\hline 20X-6, 109-134 & 157.2 & 1.42 & 0.98 & 0.58 & 0.12 & 0.64 & 8.17 & 1.52 & - & - & 0.07 & 0.55 & 1.44 & 410 & 56 & 147 \\
\hline $21 X-3,125-150$ & 164.1 & 1.47 & 1.06 & 0.57 & 0.11 & 0.59 & 9.46 & 1.79 & -25.90 & 5.96 & 0.07 & 0.55 & 1.76 & 415 & 52 & 166 \\
\hline $22 X-1,56-85$ & 170.3 & 1.74 & 0.94 & 0.87 & 0.10 & 0.76 & 9.22 & 1.23 & -25.31 & 4.60 & 0.06 & 0.51 & 2.13 & 410 & 54 & 227 \\
\hline $24 X-1,117-147$ & 176.5 & 1.91 & 1.05 & 0.93 & 0.16 & 1.05 & 6.65 & 1.00 & - & - & 0.07 & 0.72 & 1.94 & 414 & 69 & 185 \\
\hline $24 X-3,63-93$ & 178.5 & 2.04 & 1.14 & 1.03 & 0.13 & 1.29 & 8.91 & 0.89 & -25.21 & 5.37 & 0.09 & 0.65 & 1.93 & 412 & 57 & 169 \\
\hline $25 X-1,48-84$ & 179.9 & 1.73 & 1.05 & 0.83 & 0.16 & 1.16 & 6.52 & 0.91 & -25.43 & 5.16 & 0.08 & 0.59 & 1.69 & 411 & 56 & 161 \\
\hline $26 X-1,105-140$ & 183.1 & 1.49 & 0.84 & 0.79 & 0.11 & 1.30 & 7.64 & 0.64 & -25.22 & 4.18 & 0.05 & 0.44 & 1.86 & 411 & 52 & 221 \\
\hline \multicolumn{17}{|l|}{ 344-U1413C- } \\
\hline 2R-2, 119-149 & 180.5 & 1.54 & 0.89 & 0.72 & 0.11 & 1.61 & 8.02 & 0.55 & - & - & 0.06 & 0.54 & 1.90 & 408 & 61 & 213 \\
\hline $2 \mathrm{R}-5,53-83$ & 183.9 & 1.20 & 0.91 & 0.45 & 0.11 & 1.98 & 8.13 & 0.46 & -25.36 & 3.49 & 0.09 & 0.51 & 1.33 & 408 & 56 & 146 \\
\hline $3 R-1,90-122$ & 188.6 & 1.28 & 0.91 & 0.50 & 0.12 & 1.73 & 7.78 & 0.53 & -25.27 & 4.77 & 0.14 & 0.55 & 1.31 & 404 & 60 & 144 \\
\hline 5R-1, 116-146 & 208.3 & 1.66 & 0.86 & 0.93 & 0.10 & 1.56 & 9.05 & - & -24.42 & 4.33 & 0.06 & 0.51 & 1.63 & 406 & 59 & 190 \\
\hline 6R-3, 99-129 & 220.7 & 1.52 & 0.88 & 0.72 & 0.12 & 0.75 & 7.46 & 1.17 & -25.64 & 4.10 & 0.05 & 0.43 & 2.31 & 414 & 49 & 262 \\
\hline 7R-2, 77-107 & 228.8 & 1.65 & 1.06 & 0.75 & 0.12 & 0.95 & 9.06 & 1.12 & -24.75 & 5.91 & 0.08 & 0.67 & 1.68 & 413 & 63 & 158 \\
\hline 7R-6, 101-131 & 234.5 & 1.42 & 0.88 & 0.67 & 0.12 & 1.60 & 7.52 & 0.55 & - & - & 0.08 & 0.50 & 1.52 & 410 & 57 & 173 \\
\hline $8 R-4,87-117$ & 241.4 & 1.34 & 0.81 & 0.66 & 0.10 & 0.36 & 7.94 & 2.26 & -26.31 & 4.92 & 0.05 & 0.40 & 1.71 & 422 & 49 & 211 \\
\hline $10 \mathrm{R}-4,116-150$ & 260.7 & 1.83 & 0.96 & 1.01 & 0.12 & 0.71 & 7.80 & 1.36 & -24.49 & 6.03 & 0.10 & 0.55 & 1.88 & 414 & 57 & 196 \\
\hline 11R-1, 114-151 & 266.4 & 1.63 & 1.08 & 0.70 & 0.11 & 1.19 & 9.56 & 0.91 & -26.81 & 5.85 & 0.08 & 0.57 & 1.35 & 414 & 53 & 125 \\
\hline $12 \mathrm{R}-5,80-119$ & 280.1 & 1.91 & 1.30 & 0.81 & 0.13 & 0.74 & 9.77 & 1.77 & -26.60 & 5.62 & 0.08 & 0.73 & 1.75 & 418 & 56 & 135 \\
\hline $13 R-3,93-128$ & 288.4 & 1.76 & 1.22 & 0.70 & 0.13 & 0.94 & 9.31 & - & -25.74 & 5.81 & 0.08 & 0.81 & 1.67 & 417 & 66 & 137 \\
\hline 14R-4,16-61 & 299.0 & 1.50 & 1.09 & 0.60 & 0.12 & 1.60 & 8.86 & 0.68 & -25.00 & 6.18 & 0.07 & 0.59 & 1.48 & 412 & 54 & 136 \\
\hline $15 \mathrm{R}-1,65-95$ & 304.8 & 1.59 & 1.10 & 0.63 & 0.14 & 1.31 & 7.86 & 0.84 & -24.54 & 6.72 & 0.10 & 0.76 & 1.61 & 412 & 69 & 146 \\
\hline 15R-2, 103-135 & 306.1 & 1.55 & 0.99 & 0.65 & 0.12 & 2.55 & 8.18 & 0.39 & - & - & 0.07 & 0.61 & 1.81 & 411 & 62 & 183 \\
\hline 16R-3,114-150 & 317.8 & 1.91 & 1.20 & 0.82 & 0.16 & 1.64 & 7.36 & 0.73 & -23.68 & 4.73 & 0.09 & 0.92 & 1.86 & 417 & 77 & 155 \\
\hline $17 R-4,92-122$ & 328.6 & 1.82 & 1.19 & 0.77 & 0.13 & 0.97 & 9.37 & 1.22 & -24.04 & 5.13 & 0.07 & 0.79 & 2.08 & 416 & 66 & 175 \\
\hline 19R-5, 66-96 & 347.9 & 1.72 & 1.19 & 0.74 & 0.16 & 0.83 & 7.35 & 1.44 & -25.16 & 4.96 & 0.10 & 0.71 & 1.57 & 418 & 60 & 132 \\
\hline $20 \mathrm{R}-3,80-110$ & 355.8 & 1.86 & 1.19 & 0.82 & 0.13 & 0.69 & 9.15 & 1.72 & -24.93 & 5.89 & 0.07 & 0.81 & 1.59 & 422 & 68 & 134 \\
\hline $21 \mathrm{R}-4,123-153$ & 368.0 & 1.67 & 1.27 & 0.56 & 0.16 & 1.19 & 8.09 & 1.07 & -24.29 & 5.72 & 0.09 & 1.06 & 1.88 & 419 & 83 & 148 \\
\hline 22R-4, 89-119 & 377.4 & 1.79 & 1.29 & 0.62 & 0.14 & 0.87 & 9.56 & 1.48 & -23.52 & 5.88 & 0.08 & 1.09 & 2.03 & 418 & 84 & 157 \\
\hline $23 \mathrm{R}-3,122-152$ & 385.7 & 1.33 & 0.82 & 0.61 & 0.14 & 1.45 & 5.90 & 0.56 & -23.71 & 4.67 & 0.05 & 0.50 & 1.33 & 413 & 61 & 162 \\
\hline $24 R-3,112-142$ & 395.3 & 1.76 & 1.14 & 0.76 & 0.12 & 1.25 & 9.34 & 0.92 & -23.70 & 4.55 & 0.08 & 0.86 & 1.52 & 413 & 75 & 133 \\
\hline $25 R-4,122-152$ & 406.8 & - & 1.13 & 0.82 & - & 1.23 & - & 0.92 & -24.00 & 8.35 & 0.08 & 0.81 & 1.30 & 415 & 72 & 115 \\
\hline $26 \mathrm{R}-3,118-148$ & 414.5 & 1.69 & 1.24 & 0.67 & 0.12 & 1.45 & 10.00 & 0.86 & -23.81 & 6.12 & 0.08 & 0.97 & 1.52 & 425 & 78 & 123 \\
\hline $27 \mathrm{R}-2,120-150$ & 423.1 & 1.94 & 1.20 & 0.93 & 0.16 & 1.33 & 7.59 & 0.90 & -23.69 & 6.38 & 0.07 & 0.74 & 1.58 & 417 & 62 & 132 \\
\hline $28 \mathrm{R}-3,88-123$ & 433.5 & 1.23 & 0.87 & 0.47 & 0.12 & 1.45 & 7.25 & 0.60 & -23.13 & 6.07 & 0.05 & 0.49 & 1.74 & 417 & 56 & 200 \\
\hline $29 \mathrm{R}-3,100-135$ & 443.5 & 1.46 & 0.88 & 0.72 & 0.15 & 0.44 & 6.03 & 2.01 & -23.59 & 6.16 & 0.07 & 0.62 & 1.50 & 416 & 70 & 170 \\
\hline 30R-3, 96-131 & 453.1 & 1.50 & 1.01 & 0.66 & 0.11 & 0.84 & 9.62 & 1.20 & -24.10 & 6.54 & 0.06 & 0.63 & 1.39 & 417 & 62 & 138 \\
\hline
\end{tabular}


Table T1 (continued). (Continued on next page).

\begin{tabular}{|c|c|c|c|c|c|c|c|c|c|c|c|c|c|c|c|c|}
\hline $\begin{array}{l}\text { Core, section, } \\
\text { interval }(\mathrm{cm})\end{array}$ & $\begin{array}{l}\text { Depth } \\
\text { (mbsf) }\end{array}$ & $\begin{array}{c}\text { TC } \\
\text { (wt\%) }\end{array}$ & $\begin{array}{l}\text { TOC } \\
\text { (wt\%) }\end{array}$ & $\begin{array}{c}\text { TIC } \\
\text { (wt\%) }\end{array}$ & $\begin{array}{c}\mathrm{TN} \\
(\mathrm{wt} \%)\end{array}$ & $\begin{array}{c}\text { TS } \\
\text { (wt\%) }\end{array}$ & TOC/TN & TOC/TS & $\begin{array}{c}\delta^{13} \mathrm{C}_{\text {org }} \\
(\% o)\end{array}$ & $\begin{array}{c}\delta^{15} N_{\text {org }} \\
(\% o)\end{array}$ & $\begin{array}{c}\mathrm{S}_{1} \\
\text { (mg HC/g rock) }\end{array}$ & $\begin{array}{c}\mathrm{S}_{2} \\
\text { (mg HC/g rock) }\end{array}$ & $\begin{array}{c}\mathrm{S}_{3} \\
\text { (mg CO} 2 / g \text { rock) }\end{array}$ & $\begin{array}{l}T_{\max } \\
\left({ }^{\circ} \mathrm{C}\right)\end{array}$ & $\begin{array}{c}\mathrm{HI} \\
(\mathrm{mg} \mathrm{HC} / \mathrm{g} \text { TOC) }\end{array}$ & $\begin{array}{c}\mathrm{Ol} \\
(\mathrm{mg} \mathrm{CO} / \mathrm{g} \text { TOC) }\end{array}$ \\
\hline $31 \mathrm{R}-4,83-118$ & 464.3 & 1.58 & 0.91 & 0.83 & 0.11 & 1.28 & 8.35 & 0.71 & -24.55 & 6.73 & 0.05 & 0.52 & 1.53 & 416 & 57 & 168 \\
\hline $32 \mathrm{R}-5,66-101$ & 475.1 & 1.54 & 0.94 & 0.66 & 0.11 & 0.86 & 8.55 & 1.09 & -24.30 & 6.48 & 0.07 & 0.64 & 1.29 & 417 & 68 & 137 \\
\hline $33 R-5,0-30$ & 484.4 & 1.69 & 1.08 & 0.79 & 0.13 & 1.03 & 8.37 & 1.05 & -24.29 & 5.79 & 0.06 & 0.78 & 1.68 & 415 & 72 & 156 \\
\hline $34 \mathrm{R}-2,87-122$ & 490.8 & 1.24 & 0.87 & 0.45 & 0.12 & 0.86 & 7.37 & 1.01 & -23.57 & 7.19 & 0.06 & 0.64 & 1.16 & 420 & 74 & 133 \\
\hline $35 \mathrm{R}-3,94-129$ & 502.0 & 0.98 & 0.66 & 0.36 & 0.10 & 1.39 & 6.73 & 0.47 & -25.68 & 6.86 & 0.05 & 0.35 & 1.05 & 414 & 53 & 159 \\
\hline $36 \mathrm{R}-5,62-97$ & 514.1 & 0.89 & 0.67 & 0.32 & 0.12 & 1.07 & 5.40 & 0.63 & -25.36 & 7.03 & 0.04 & 0.37 & 1.09 & 423 & 55 & 163 \\
\hline $37 R-5,61-96$ & 523.6 & 0.94 & 0.41 & 0.52 & 0.09 & 0.77 & 4.71 & 0.53 & -26.45 & 6.70 & 0.03 & 0.20 & 1.10 & 412 & 49 & 268 \\
\hline 38R-4, 117-152 & 532.9 & 0.97 & 0.63 & 0.38 & 0.12 & 0.56 & 5.25 & 1.12 & -26.88 & 6.64 & 0.04 & 0.36 & 0.91 & 417 & 57 & 144 \\
\hline $39 R-4,116-151$ & 542.3 & 0.17 & 0.55 & 0.65 & 0.07 & 0.46 & 7.43 & 1.19 & -26.87 & 8.20 & 0.03 & 0.29 & 1.21 & 422 & 53 & 220 \\
\hline 40R-6, 97-132 & 554.6 & 0.99 & 0.73 & 0.32 & 0.12 & 0.98 & 5.89 & 0.74 & -26.40 & 7.93 & 0.04 & 0.40 & 1.01 & 414 & 55 & 138 \\
\hline $41 \mathrm{R}-2,116-151$ & 559.0 & 0.92 & 0.63 & 0.37 & 0.09 & 1.04 & 6.85 & 0.60 & - & - & 0.04 & 0.30 & 1.06 & 413 & 48 & 168 \\
\hline $42 \mathrm{R}-2,56-91$ & 568.1 & 0.72 & 0.53 & 0.23 & 0.10 & 1.27 & 5.35 & 0.42 & -26.48 & 8.29 & 0.04 & 0.28 & 0.68 & 416 & 53 & 128 \\
\hline $43 R-2,73-105$ & 577.7 & 0.71 & 1.70 & 0.37 & 0.09 & 1.09 & 19.10 & 1.56 & -24.65 & 6.98 & 0.30 & 2.14 & 3.33 & 418 & 126 & 196 \\
\hline \multicolumn{17}{|l|}{ 344-U1414A- } \\
\hline $1 \mathrm{H}-1,51-61$ & 0.6 & 1.91 & 1.69 & 0.38 & 0.20 & 0.17 & 8.33 & 9.67 & - & - & 0.30 & 2.21 & 3.24 & 412 & 131 & 192 \\
\hline $2 \mathrm{H}-1,131-141$ & 3.3 & 2.41 & 1.48 & 0.93 & 0.17 & 0.53 & 8.92 & 2.78 & - & - & 0.24 & 1.97 & 3.16 & 419 & 133 & 214 \\
\hline $2 \mathrm{H}-3,131-141$ & 6.1 & 2.05 & 1.45 & 0.85 & 0.15 & 0.13 & 9.80 & 11.13 & - & - & 0.21 & 1.49 & 2.64 & 421 & 103 & 182 \\
\hline $2 \mathrm{H}-5,131-141$ & 8.9 & 1.89 & 1.70 & 0.49 & 0.19 & 0.67 & 8.85 & 2.53 & - & - & 0.25 & 1.79 & 2.90 & 421 & 105 & 171 \\
\hline $3 \mathrm{H}-2,131-141$ & 14.2 & 2.27 & 1.80 & 0.65 & 0.18 & 0.59 & 9.78 & 3.06 & - & - & 0.28 & 2.20 & 3.26 & 423 & 122 & 181 \\
\hline $3 \mathrm{H}-5,131-141$ & 18.4 & 1.78 & 1.71 & 0.30 & 0.18 & 0.99 & 9.55 & 1.73 & - & - & 0.29 & 2.16 & 2.57 & 420 & 126 & 150 \\
\hline $4 \mathrm{H}-2,131-141$ & 23.7 & 1.70 & 1.66 & 0.26 & 0.16 & 0.58 & 10.12 & 2.87 & - & - & 0.27 & 1.93 & 2.33 & 418 & 116 & 140 \\
\hline $4 \mathrm{H}-5,132-142$ & 27.9 & 2.33 & 1.91 & 0.65 & 0.17 & 1.01 & 11.24 & 1.89 & - & - & 0.28 & 2.10 & 3.26 & 420 & 110 & 171 \\
\hline $5 \mathrm{H}-2,131-141$ & 33.2 & 2.44 & 2.00 & 0.68 & 0.18 & 0.93 & 10.93 & 2.15 & - & - & 0.33 & 2.62 & 3.28 & 422 & 131 & 164 \\
\hline $5 \mathrm{H}-5,131-141$ & 37.4 & 1.82 & 1.52 & 0.45 & 0.16 & 1.15 & 9.68 & 1.32 & - & - & 0.33 & 1.74 & 2.64 & 418 & 114 & 174 \\
\hline $6 \mathrm{H}-2,135-145$ & 42.8 & 2.08 & 1.31 & 0.88 & 0.14 & 2.34 & 9.63 & 0.56 & - & - & 0.25 & 1.28 & 2.95 & 414 & 98 & 225 \\
\hline $6 \mathrm{H}-5,135-145$ & 47.1 & 2.76 & 1.83 & 1.19 & 0.16 & 2.50 & 11.16 & 0.73 & - & - & 0.25 & 2.21 & 3.29 & 421 & 121 & 180 \\
\hline $7 \mathrm{H}-2,140-150$ & 52.4 & 1.87 & 1.24 & 0.77 & 0.13 & 2.09 & 9.61 & 0.59 & - & - & 0.20 & 1.04 & 2.74 & 413 & 84 & 221 \\
\hline 7H-5, 140-150 & 56.9 & 2.56 & 1.76 & 1.04 & 0.15 & 2.30 & 11.73 & 0.76 & - & - & 0.23 & 1.90 & 3.19 & 422 & 108 & 181 \\
\hline $8 \mathrm{H}-2,140-150$ & 61.9 & 1.43 & 1.18 & 0.40 & 0.13 & 2.14 & 9.29 & 0.55 & - & - & 0.20 & 0.93 & 2.38 & 415 & 79 & 202 \\
\hline $9 \mathrm{H}-2,140-150$ & 71.4 & 2.71 & 1.71 & 1.18 & 0.15 & 2.40 & 11.10 & 0.71 & - & - & 0.31 & 1.71 & 3.37 & 420 & 100 & 197 \\
\hline $9 \mathrm{H}-5,140-150$ & 75.9 & 1.51 & 1.23 & 0.46 & 0.13 & 1.99 & 9.76 & 0.62 & - & - & 0.21 & 0.93 & 2.70 & 418 & 76 & 220 \\
\hline $10 \mathrm{H}-2,140-150$ & 80.9 & 1.45 & 1.09 & 0.53 & 0.13 & 0.89 & 8.38 & 1.22 & - & - & 0.19 & 0.76 & 2.49 & 414 & 70 & 228 \\
\hline $10 \mathrm{H}-5,140-150$ & 85.4 & 2.32 & 1.55 & 0.98 & 0.13 & 1.34 & 11.83 & 1.16 & - & - & 0.16 & 1.29 & 3.30 & 424 & 83 & 213 \\
\hline $11 \mathrm{H}-2,140-150$ & 90.4 & 1.89 & 1.16 & 0.92 & 0.12 & 2.01 & 9.59 & 0.58 & - & - & 0.19 & 0.67 & 2.57 & 411 & 58 & 222 \\
\hline $11 \mathrm{H}-5,140-150$ & 94.9 & 1.05 & 0.88 & 0.29 & 0.11 & 1.26 & 7.93 & 0.70 & - & - & 0.16 & 0.66 & 1.75 & 402 & 75 & 199 \\
\hline $12 \mathrm{H}-2,140-150$ & 99.9 & 1.60 & 1.17 & 0.60 & 0.13 & 0.51 & 8.80 & 2.29 & - & - & 0.21 & 0.96 & 2.19 & 413 & 82 & 187 \\
\hline $12 \mathrm{H}-5,140-150$ & 104.4 & 1.98 & 1.17 & 0.98 & 0.12 & 3.56 & 10.17 & 0.33 & - & - & 0.18 & 0.80 & 2.54 & 411 & 68 & 217 \\
\hline $13 \mathrm{H}-2,140-150$ & 109.4 & 1.48 & 0.82 & 0.74 & 0.09 & 1.67 & 8.72 & 0.49 & - & - & 0.15 & 0.56 & 1.89 & 408 & 68 & 230 \\
\hline $13 \mathrm{H}-5,140-150$ & 113.9 & 1.11 & 0.91 & 0.30 & 0.12 & 1.01 & 7.84 & 0.90 & - & - & 0.17 & 0.79 & 1.30 & 416 & 87 & 143 \\
\hline $14 \mathrm{H}-2,140-150$ & 118.9 & 2.31 & 1.04 & 1.50 & 0.11 & 1.35 & 9.54 & 0.77 & - & - & 0.17 & 0.69 & 2.06 & 412 & 66 & 198 \\
\hline $14 \mathrm{H}-5,135-150$ & 123.3 & 2.95 & 0.74 & 2.45 & 0.09 & 0.19 & 8.13 & 3.85 & - & - & 0.11 & 0.49 & 2.21 & 412 & 66 & 299 \\
\hline $15 \mathrm{H}-2,140-150$ & 128.4 & 3.17 & 0.82 & 2.54 & 0.10 & 0.71 & 8.45 & 1.16 & - & - & 0.13 & 0.50 & 2.10 & 413 & 61 & 256 \\
\hline $15 \mathrm{H}-5,140-150$ & 132.9 & 2.01 & 0.92 & 1.26 & 0.11 & 0.05 & 8.52 & 19.42 & - & - & 0.19 & 0.84 & 2.20 & 414 & 91 & 239 \\
\hline $16 \mathrm{H}-2,110-120$ & 137.6 & 3.82 & 1.00 & 3.04 & 0.11 & 0.14 & 9.52 & 7.23 & - & - & 0.07 & 0.68 & 2.69 & 405 & 68 & 269 \\
\hline $16 \mathrm{H}-5,140-150$ & 142.1 & 4.01 & 0.77 & 3.52 & 0.08 & - & 9.51 & - & - & - & 0.10 & 0.44 & 2.66 & 404 & 57 & 345 \\
\hline $18 \mathrm{H}-5,130-150$ & 159.4 & 5.49 & 0.60 & 5.16 & 0.06 & 0.54 & 9.52 & 1.12 & - & - & 0.11 & 0.32 & 1.94 & 377 & 53 & 323 \\
\hline $19 \mathrm{H}-3,135-150$ & 166.3 & 5.35 & 0.70 & 5.02 & 0.09 & 0.32 & 8.14 & 2.17 & - & - & 0.04 & 0.36 & 2.00 & 408 & 51 & 286 \\
\hline $19 \mathrm{H}-6,135-150$ & 170.7 & 5.50 & 0.53 & 5.34 & 0.05 & 1.24 & 10.82 & 0.43 & - & - & 0.04 & 0.19 & 2.05 & 360 & 36 & 387 \\
\hline $20 \mathrm{H}-2,135-150$ & 174.5 & 6.91 & 0.41 & 7.10 & 0.05 & 0.27 & 7.74 & 1.50 & - & - & 0.07 & 0.24 & 1.65 & 364 & 59 & 402 \\
\hline $21 \mathrm{H}-3,135-150$ & 185.5 & 7.58 & 0.32 & 8.02 & 0.04 & 0.51 & 8.89 & 0.62 & - & - & 0.03 & 0.18 & 1.41 & 367 & 56 & 441 \\
\hline $22 \mathrm{H}-6,70-85$ & 198.9 & 7.47 & 0.35 & 7.76 & 0.04 & 0.14 & 9.72 & 2.56 & - & - & 0.05 & 0.26 & 1.25 & 416 & 74 & 357 \\
\hline
\end{tabular}


Table T1 (continued).

\begin{tabular}{|c|c|c|c|c|c|c|c|c|c|c|c|c|c|c|c|c|}
\hline $\begin{array}{l}\text { Core, section, } \\
\text { interval }(\mathrm{cm})\end{array}$ & $\begin{array}{l}\text { Depth } \\
\text { (mbsf) }\end{array}$ & $\begin{array}{c}\text { TC } \\
\text { (wt\%) }\end{array}$ & $\begin{array}{l}\text { TOC } \\
\text { (wt\%) }\end{array}$ & $\begin{array}{c}\text { TIC } \\
(w t \%)\end{array}$ & $\begin{array}{c}\mathrm{TN} \\
(\mathrm{wt} \%)\end{array}$ & $\begin{array}{c}\text { TS } \\
\text { (wt\%) }\end{array}$ & $\mathrm{TOC} / \mathrm{TN}$ & $\mathrm{TOC} / \mathrm{TS}$ & $\begin{array}{c}\delta^{13} C_{\text {org }} \\
(\% o)\end{array}$ & $\begin{array}{l}\delta^{15} \mathrm{~N}_{\text {org }} \\
(\% 0)\end{array}$ & $\begin{array}{c}\mathrm{S}_{1} \\
\text { (mg HC/g rock) }\end{array}$ & $\begin{array}{c}\mathrm{S}_{2} \\
\text { (mg HC/g rock) }\end{array}$ & $\begin{array}{c}\mathrm{S}_{3} \\
\text { (mg CO} \\
2 / g \text { rock) }\end{array}$ & $\begin{array}{l}T_{\max } \\
\left({ }^{\circ} \mathrm{C}\right)\end{array}$ & $\begin{array}{c}\mathrm{HI} \\
(\mathrm{mg} \mathrm{HC} / \mathrm{g} \text { TOC) }\end{array}$ & $\begin{array}{c}\mathrm{Ol} \\
(\mathrm{mg} \mathrm{CO} / \mathrm{g} \text { TOC) }\end{array}$ \\
\hline $23 X-2,130-150$ & 203.0 & 8.92 & 0.35 & 9.72 & 0.04 & 0.18 & 9.72 & 1.95 & - & - & 0.03 & 0.26 & 1.19 & 417 & 74 & 340 \\
\hline $23 X-5,130-150$ & 207.5 & 8.86 & 0.32 & 9.75 & 0.03 & 0.46 & 12.31 & 0.70 & - & - & 0.03 & 0.25 & 1.18 & 422 & 78 & 369 \\
\hline $24 X-4,94-114$ & 214.9 & 4.87 & 0.57 & 4.74 & 0.04 & 0.62 & 13.26 & 0.91 & - & - & 0.04 & 0.20 & 2.05 & 410 & 35 & 360 \\
\hline $24 X-6,130-150$ & 217.9 & 5.10 & 0.64 & 4.73 & 0.04 & 1.02 & 15.24 & 0.63 & - & - & 0.04 & 0.20 & 1.96 & 405 & 31 & 306 \\
\hline $25 X-2,112-132$ & 222.0 & 6.97 & 0.74 & 7.01 & 0.04 & 0.40 & 16.82 & 1.86 & - & - & 0.04 & 0.46 & 1.63 & 426 & 62 & 220 \\
\hline $25 X-5,130-150$ & 226.5 & 2.95 & 1.05 & 2.04 & 0.08 & 1.67 & 13.46 & 0.63 & - & - & 0.06 & 1.12 & 1.79 & 428 & 107 & 170 \\
\hline $26 \mathrm{X}-2,130-150$ & 231.8 & 4.77 & 0.87 & 4.19 & 0.05 & - & 16.11 & - & - & - & 0.05 & 0.33 & 1.85 & 417 & 38 & 213 \\
\hline $26 X-5,130-150$ & 236.3 & 6.49 & 0.63 & 6.36 & 0.04 & 0.56 & 15.37 & 1.13 & - & - & 0.04 & 0.23 & 1.72 & 415 & 37 & 273 \\
\hline $27 X-3,120-150$ & 242.9 & 5.12 & 0.96 & 4.46 & 0.07 & 0.57 & 14.12 & 1.69 & - & - & 0.07 & 0.62 & 1.82 & 421 & 65 & 190 \\
\hline $28 X-4,67-97$ & 252.9 & 8.42 & 0.34 & 8.91 & 0.03 & 0.15 & 12.59 & 2.29 & - & - & 0.02 & 0.10 & 0.98 & 409 & 29 & 288 \\
\hline $29 X-2,72-102$ & 260.2 & 8.03 & 0.37 & 8.77 & 0.03 & 0.23 & 11.94 & 1.58 & - & - & 0.03 & 0.21 & 1.02 & 421 & 57 & 276 \\
\hline $30 \times-2,79-109$ & 269.4 & 9.66 & 0.18 & 10.81 & 0.02 & 0.11 & 9.47 & 1.59 & - & - & 0.02 & 0.11 & 0.82 & 421 & 61 & 456 \\
\hline $30 X-4,65-95$ & 271.1 & 9.61 & 0.20 & 10.70 & 0.01 & 0.12 & 14.29 & 1.66 & - & - & 0.02 & 0.11 & 0.79 & 422 & 55 & 395 \\
\hline $31 X-2,65-100$ & 279.0 & 9.93 & 0.24 & 10.99 & 0.02 & 0.17 & 10.00 & 1.40 & - & - & 0.01 & 0.11 & 0.74 & 421 & 46 & 308 \\
\hline $32 X-2,91-121$ & 288.8 & 9.28 & 0.38 & 10.22 & 0.02 & - & 21.11 & - & - & - & 0.02 & 0.12 & 0.76 & 425 & 32 & 200 \\
\hline $34 \mathrm{X}-1,0-14$ & 302.2 & 8.30 & 0.64 & 8.40 & 0.03 & 0.19 & 25.60 & 3.44 & - & - & 0.03 & 0.32 & 1.19 & 424 & 50 & 186 \\
\hline 36R-2, 67-92 & 314.0 & 7.72 & 0.80 & 7.81 & 0.04 & 0.04 & 21.62 & 18.03 & - & - & 0.02 & 0.23 & 0.61 & 423 & 29 & 76 \\
\hline $37 \mathrm{R}-3,74-103$ & 319.5 & 8.14 & 0.72 & 7.57 & 0.05 & 0.88 & 15.65 & 0.82 & - & - & 0.04 & 0.31 & 0.96 & 426 & 43 & 133 \\
\hline $38 \mathrm{R}-1,123-145$ & 327.4 & 7.38 & 0.76 & 6.70 & 0.03 & 0.39 & 22.35 & 1.94 & - & - & 0.04 & 0.39 & 0.92 & 428 & 51 & 121 \\
\hline $38 \mathrm{R}-2,66-102$ & 328.4 & 8.77 & 0.87 & 8.60 & 0.04 & 0.44 & 22.31 & 1.98 & - & - & 0.02 & 0.79 & 0.93 & 430 & 91 & 107 \\
\hline $39 \mathrm{R}-1,80-94$ & 336.7 & 6.86 & 1.22 & 6.75 & 0.03 & 0.52 & 38.13 & 2.36 & - & - & 0.03 & 0.83 & 0.76 & 425 & 68 & 62 \\
\hline 39R-1, 94-111 & 336.8 & 7.97 & 1.15 & 8.10 & 0.05 & 0.09 & 21.70 & 12.27 & - & - & 0.26 & 1.28 & 0.73 & 429 & 111 & 63 \\
\hline
\end{tabular}

$\mathrm{TC}=$ total carbon, $\mathrm{TOC}=$ total organic carbon, $\mathrm{TIC}=$ total inorganic carbon, $\mathrm{TN}=$ total nitrogen, $\mathrm{TS}=$ total sulfur, $T_{\max }=$ maximum $\mathrm{S}_{2}$ temperature, $\mathrm{S}_{1}=$ first pyrogram peak under low temperature $\left(<300^{\circ} \mathrm{C}\right), S_{2}=$ second pyrogram temperature peak, $S_{3}=$ third pyrogram temperature peak, $\mathrm{HI}=$ hydrogen index, $\mathrm{OI}=$ oxygen index. $-=$ no measurement. 